\title{
LES Prediction of Flow and Acoustic Field of a Coaxial Jet
}

\author{
Niklas Andersson* ${ }^{*}$ Lars-Erik Eriksson ${ }^{\dagger}$ and Lars Davidson ${ }^{\dagger}$ \\ Division of Fluid Dynamics, Department of Applied Mechanics, Chalmers University of Technology, \\ SE-412 96 Göteborg, Sweden
}

\begin{abstract}
A compressible high-subsonic coaxial jet has been simulated using large-eddy simulation (LES). The acoustic field was extended to the far field using Kirchhoff surface integration. The jet Mach number based on the local speed of sound is approximately 0.9 for both the primary and secondary stream. The static temperature in the primary stream is three times that of the secondary stream.

In order to resolve the acoustic field, it is desirable to have a computational domain with a rather large radial extent and a mesh that is relatively fine even in the far-field regions. Furthermore, the mesh should be as equidistant as possible so as to minimize the introduction of numerical errors. In order to keep the number of cells down, the computational domain was divided into three regions: a well resolved near-wall LES region, a medium-resolution LES region optimized for propagation of acoustic waves, and a coarse LES region. Over the interfaces between these regions, the number of cells is increased by factor two in each direction. Special treatment of the interfaces between the regions is utilized in order to minimize undesirable numerical errors.

The radial extent of the computational domain increases downstream such that the flow in the outer boundary region can be assumed to be irrotational and axisymmetric. Hence, the flow outside the three-dimensional computational domain can be represented by a less expensive two-dimensional axisymmetric calculation. The interface between the full 3D LES region and the $2 D$ region is based on azimuthally averaged quantities and acts as an absorbing boundary condition.

The Favre-filtered Navier-Stokes equations were solved using a finite-volume method solver with a low-dissipation third-order upwind scheme for the convective fluxes, a secondorder centered difference approach for the viscous fluxes and a three-stage second-order Runge-Kutta technique in time. The computational domain was discretized using a blockstructured boundary-fitted mesh with approximately $2.0 \times 10^{7}$ nodes. The calculations were performed on a parallel computer, using message-passing interface (MPI). A compressible form of Smagorinsky's subgrid-scale model was used to compute the subgrid-scale stresses.
\end{abstract}

${ }^{*}$ Ph.D. Student, Division of Fluid Dynamics, Department of Applied Mechanics, Chalmers University of Technology, SE-412 96 Göteborg, Sweden

†Professor, Division of Fluid Dynamics, Department of Applied Mechanics, Chalmers University of Technology, SE-412 96 Göteborg, Sweden

Copyright (c) 2005 by Niklas Andersson, Lars-Erik Eriksson \& Lars Davidson. Published by the American Institute of Aeronautics and Astronautics, Inc. with permission. 


\section{Nomenclature}

Latin symbols

$\begin{array}{ll}c & \text { speed of sound } \\ C_{p} & \text { specific heat at constant pressure } \\ C_{R}, C_{I} & \text { Smagorinsky model coefficients } \\ D & \text { nozzle diameter } \\ e & \text { energy } \\ k & \text { kinetic energy } \\ M & \text { Mach number } \\ p & \text { pressure } \\ P r & \text { Prandtl number } \\ Q & \text { state vector } \\ q_{j} & \text { energy diffusion vector } \\ \mathcal{R} & \text { correlation } \\ r & \text { radial coordinate or distance from source to observer } \\ R e_{D} & \text { Reynolds number } \\ S_{i j} & \text { strain rate tensor } \\ T & \text { temperature } \\ t & \text { time } \\ U & \text { velocity } \\ u_{i} & \text { Cartesian components of velocity vector } \\ \mathbf{x} & \text { flow location } \\ x_{i} & \text { Cartesian coordinate vector component } \\ \mathbf{y} & \text { far-field location }\end{array}$

Greek symbols

$\begin{array}{ll}\Delta & \text { filter width } \\ \delta_{i j} & \text { Kronecker delta } \\ \gamma & \text { gas constant } \\ \mu & \text { dynamic viscosity } \\ \rho & \text { density } \\ \sigma_{i j} & \text { viscous stress tensor } \\ \tau & \text { temporal separation } \\ \tau_{i j} & \text { subgrid-scale stress tensor } \\ \tau_{r} & \text { retarded time } \\ \xi & \text { spatial separation } \\ \text { Subscripts } & \\ \infty & \text { ambient condition } \\ j & \text { jet-exit condition } \\ p & \text { primary stream } \\ r m s & \text { root-mean-square } \\ s & \text { secondary stream } \\ t & \text { turbulent quantity } \\ \text { Superscripts } & \\ - & \text { spatially filtered quantity } \\ \zeta & \text { resolved fluctuation } \\ \sim & \text { spatially Favre-filtered quantity }\end{array}$


Symbols

$\langle\ldots\rangle_{\theta} \quad$ circumferentially averaged quantity

$\langle\ldots\rangle_{t} \quad$ time-averaged quantity

\section{Introduction}

QTRICTER noise regulation for near-ground operations has made noise reduction in commercial airScraft a topic of growing interest in the aerospace industry. Despite progress in the development of CFD solvers, most of the noise prediction methods currently in use in industry are correlations based on empirical databases. The reason for this is the extreme demands for numerical accuracy in computational aeroacoustic (CAA) methods that place high demands on computational resources. However, to be able to account for changes in the flow by new noise reduction techniques or to predict differences in radiated sound in different engine concepts at an early design stage, more explicit approaches are required. With continuously increasing computer capacity and with the possibility to carry out parallel computations on PC clusters, computational aeroacoustics has now, to some extent, become feasible for industrial use.

Large-eddy simulation (LES) has recently been established as a numerical approach for the simulation of turbulent flows in situations in which detailed information on turbulence characteristics and flow physics is desirable and direct numerical simulation (DNS) is far too expensive. To be able to accurately predict flow-induced noise, flow simulation techniques of high accuracy have to be used. Using a compressible solver the acoustic pressure signal in far-field locations can be obtained directly from the flow simulation. This, however, leads to extremely expensive calculations since the mesh has to be fine enough through out the domain to ensure that acoustic waves are not damped by the numerical dissipation introduced by the numerical method. The calculation costs can be decreased by the use of a hybrid approach in which the hydrodynamic jet flowfield is obtained using DNS or LES and the far-field acoustics is obtained using an integral method, e.g. Kirchhoff ${ }^{1}$ surface integration or the formulation by Ffowcs Williams \& Hawkings, ${ }^{2}$ see for example Freund et al. ${ }^{3}$ and Uzun et al. ${ }^{4,5}$ In a jet simulation the major part of the cells are often clustered in the hydrodynamic jet region so as to resolve turbulence structures in the jet. Thus using a hybrid approach like the ones mentioned above might, if the mesh is not as fine in the far field, give better results than a direct method since the cut-off frequency is higher in the integration surface region than in the far field. ${ }^{4,5}$ Andersson et al. ${ }^{6,7,8}$ used large-eddy simulation to study the flow and radiated sound of a subsonic (Mach 0.75) single-stream jet at different heating conditions. The acoustic field was extracted to the far field using Kirchhoff surface integration. The Reynolds number in that simulation was $R e_{D}=5.0 \times 10^{4}$. Results were found to be in generally good agreement with measurements although the Reynolds number was significantly higher in the experiments. In the present work the LES/Kirchhoff approach has been applied to a coaxial jet configuration. The work has been done as part of the EU 6th framework programme CoJeN ${ }^{\mathrm{a}}$, an European effort to gain understanding of the physics behind noise generation in coaxial jets.

The objective of CoJeN is to develop and validate prediction tools that can be used by the aerospace industry to assess and optimize jet noise prediction techniques. The aim is to provide design tools that can be used to develop low noise nozzles for high bypass ratio (HBR) engines. For prediction techniques to be useful to industry, the methods must cope with realistic jet flows such as coaxial jet configurations with high velocities, significant velocity and temperature gradients, and arbitrary nozzle geometries. LES is currently not feasible for industrial design use because of long turnover times and a restriction to fairly simple geometries. However, the results of a detailed LES can be useful in the development of

\footnotetext{
${ }^{\mathrm{a}}$ Computation of Coaxial Jet Noise, contract number: AST3-CT-2003-502790
} 
tools for industrial use. For example, higher-order statistics, such as two-point space-time correlations, can be evaluated.

LES and DNS have been used for jet flow applications in a number of investigations. These primarily study jets at moderate Reynolds number due to the high computational costs of making simulations of a high Reynolds number jet. Many of these studies have been carried out to predict jet noise. However, as jet flows are free shear flows frequently occurring in both nature and industrial applications, it is interesting to study the jet in itself. Some studies are thus pure investigations of flow phenomena.

The feasibility of using LES for both the flowfield and the radiated sound from a high-subsonic $6.5 \times 10^{4}$ Reynolds number jet has been discussed by Bogey et al. ${ }^{9,10,11}$ In Refs. $(9,10)$, the acoustic field was obtained directly from the flow simulation. Noise generation mechanisms were found to be relatively independent of the Reynolds number. In Ref. (11) Lighthill's ${ }^{12}$ acoustic analogy was used in combination with compressible LES to obtain the acoustic field. Bogey \& Bailly ${ }^{13}$ investigated the effects of inflow conditions on the flowfield and the radiated sound of a high Reynolds number, $R e_{D}=4.0 \times 10^{5}$, Mach 0.9 jet. Both the flow development and the emitted sound were shown to depend appreciably on inlet parameters. Bogey \& Bailly ${ }^{14}$ investigated the effects of Mach and Reynolds number on subsonic jet noise. Bodony et al. ${ }^{15}$ investigated the changes in jet mean field and far-field sound when simulating jets with different temperatures and Mach numbers. Results were found to be in agreement with published data. ${ }^{16}$ Freund ${ }^{17}$ used DNS to investigate sources of sound in a Mach 0.9 jet at a Reynolds number of $R e_{D}=3.6 \times 10^{3}$. In this work the part of the Lighthill source that may radiate to the far field was isolated using Fourier methods. It was found that the peak of the radiating source coincides neither with the peak of the total source nor the peak of turbulence kinetic energy. Shur et al..$^{18}$ made simulations of a cold Mach 0.9 jet at a Reynolds number of $1.0 \times 10^{4}$. Radiated sound was successfully predicted using Ffowcs Williams \& Hawkings ${ }^{2}$ surface integral formulation. The simulation was made using only $5.0 \times 10^{5}$ cells. This work was done with the monotone-integrated LES (MILES) approach where the subgrid-scale model is essentially replaced by the numerical dissipation of the numerical method. Zhao et al. ${ }^{19}$ made an LES of a Mach 0.9 jet at Reynolds number $3.6 \times 10^{3}$ and a jet at Mach 0.4 and a Reynolds number of $5.0 \times 10^{3}$. In this study, radiated sound was obtained both directly from the LES and by using Kirchhoff surface integration. The effect on the radiated sound of the subgrid-scale model was investigated. It was found that using a mixed subgrid-scale model resulted in both higher turbulence levels and sound levels. Uzun et al. ${ }^{4,5}$ made LES of a high-subsonic (Mach 0.9) single-stream jet at a Reynolds number of $4.0 \times 10^{5}$. Sound pressure levels obtained using surface integral methods, e.g. Kirchhoff and Ffowcs William \& Hawkings, were compared to results obtained using a volume integral method (Lighthill's acoustic analogy). The results obtained using the surface approaches were found to be comparable with the results obtained using Lighthill integration.

\section{Overview of the Present Study}

A high-subsonic coaxial jet with heated primary stream is studied. The static temperature in the primary stream (core flow) is roughly three times that of the secondary stream (bypass flow), leading to large temperature gradients in the shear layer between the two streams. The jet Mach number, $M_{j}=U_{j} / c_{j}$, is approximately 0.9 for both streams. The jet is expanded into stagnant surroundings and the temperature of the secondary stream is equal to the ambient temperature. Table 1 gives more detail on the flow properties for the simulated coaxial jet. In Figure (1), the nozzle geometry included in the computational domain is depicted: a (3/4) cowl nozzle with a center body.

No experimental data for validation of the LES predictions are available at this stage in the project. At a later stage, single-point multi-component statistics and spatial correlations will be available for validation of the predicted flow data, and the predicted acoustic signature will be evaluated using far-field acoustic measurements. 
Table 1. Flow properties

Jet flow conditions (primary (p) and secondary flow (s))

$\begin{array}{llll}U_{p} & m s^{-1} & 480.7 & \text { Fully-expanded jet velocity in the primary stream }\end{array}$

$U_{s} \quad m s^{-1} \quad 306.8 \quad$ Fully-expanded jet velocity in the secondary stream

$M_{p} \quad 0.861 \quad$ Mach number $\left(U_{j} / c_{j}\right)$ in the primary stream

$M_{s} \quad 0.902 \quad$ Mach number $\left(U_{j} / c_{j}\right)$ in the secondary stream

$\begin{array}{llll}T_{p} & K & 775.6 \quad \text { Static temperature in primary stream }\end{array}$

$T_{s} \quad K \quad 288.14 \quad$ Static temperature in secondary stream

$T_{0 p} \quad K \quad 890.6 \quad$ Total temperature in primary stream

$\begin{array}{llll}T_{0 s} & K & 335.0 & \text { Total temperature in secondary stream }\end{array}$

\section{Ambient conditions}

$\begin{array}{lll}P_{\infty} & P a & 101325 \\ \rho_{\infty} & \mathrm{kgm}^{-3} & 1.22556 \\ c_{\infty} & \mathrm{ms}^{-1} & 340.174 \\ U_{\infty} & \mathrm{ms}^{-1} & 0.0 \\ T_{\infty} & K & 288.14\end{array}$

Nozzle geometry

$\begin{array}{lll}D_{p} & m & 0.137\end{array}$

$\begin{array}{lll}D_{s} & m & 0.275\end{array}$

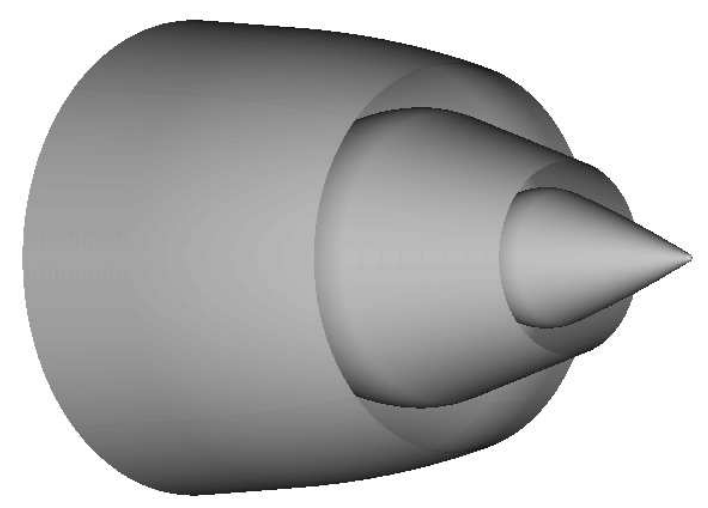

Figure 1. The CoJeN short cowl nozzle 


\section{Governing equations}

The equations solved are the spatially Favre-filtered continuity, momentum and energy equations,

$$
\begin{gathered}
\frac{\partial \bar{\rho}}{\partial t}+\frac{\partial\left(\bar{\rho} \tilde{u}_{i}\right)}{\partial x_{i}}=0 \\
\frac{\partial\left(\bar{\rho} \tilde{u}_{i}\right)}{\partial t}+\frac{\partial\left(\bar{\rho} \tilde{u}_{i} \tilde{u}_{j}\right)}{\partial x_{j}}=-\frac{\partial \bar{p}}{\partial x_{i}}+\frac{\partial \bar{\sigma}_{i j}}{\partial x_{j}}+\frac{\partial \tau_{i j}}{\partial x_{j}} \\
\frac{\partial\left(\bar{\rho} \tilde{e}_{0}\right)}{\partial t}+\frac{\partial\left(\bar{\rho} \tilde{e}_{0} \tilde{u}_{j}\right)}{\partial x_{j}}=-\frac{\partial \bar{p} \tilde{u}_{j}}{\partial x_{j}}+ \\
+\frac{\partial}{\partial x_{j}}\left(C_{p} \frac{\mu}{P r} \frac{\partial \tilde{T}}{\partial x_{j}}+q_{j}\right)+\frac{\partial}{\partial x_{j}}\left(\tilde{u}_{i}\left(\bar{\sigma}_{i j}+\tau_{i j}\right)\right)
\end{gathered}
$$

where $\bar{\sigma}_{i j}$ and $\tau_{i j}$ are the Favre-filtered viscous stress tensor and subgrid-scale viscous stress tensor, respectively. These are here defined as

$$
\begin{gathered}
\bar{\sigma}_{i j}=\mu\left(2 \tilde{S}_{i j}-\frac{2}{3} \tilde{S}_{m m} \delta_{i j}\right) \\
\tau_{i j}=\mu_{t}\left(2 \tilde{S}_{i j}-\frac{2}{3} \tilde{S}_{m m} \delta_{i j}\right)-\frac{2}{3} \bar{\rho} k^{S G S} \delta_{i j}
\end{gathered}
$$

where $k^{S G S}$ is the subgrid-scale kinetic energy

$$
k^{S G S}=C_{I} \Delta^{2} \tilde{S}_{m n} \tilde{S}_{m n}
$$

$\mu_{t}$ the subgrid-scale dynamic viscosity

$$
\mu_{t}=C_{R} \bar{\rho} \Delta^{2} \sqrt{\tilde{S}_{m n} \tilde{S}_{m n}}
$$

and $\tilde{S}_{i j}$ is the Favre-filtered strain rate tensor given by,

$$
\tilde{S}_{i j}=\frac{1}{2}\left(\frac{\partial \tilde{u}_{i}}{\partial x_{j}}+\frac{\partial \tilde{u}_{j}}{\partial x_{i}}\right)
$$

The subgrid heat flux appearing in the Favre-filtered energy equation is modeled using a temperature gradient approach

$$
q_{j}=C_{p} \frac{\mu_{t}}{\operatorname{Pr}_{t}} \frac{\partial \tilde{T}}{\partial x_{j}}
$$

The filter-width in Eqs. (6) and (7) is a representative local grid cell width. In the present work both $\Delta=\left(\Delta_{1} \Delta_{2} \Delta_{3}\right)^{1 / 3}$ and $\Delta=\min \left(\Delta_{1}, \Delta_{2}, \Delta_{3}\right)$ have been used. The subgrid-scale model used in this work is the Smagorinsky part of the model proposed by Erlebacher et al. ${ }^{20}$ for compressible flows. Constants $C_{R}$ and $C_{I}$ that appear in Eqs. (6) and (7) are the Smagorinsky model constants, of which the latter is a compressibility correction constant. These are here given by

$$
\left\{\begin{array}{l}
C_{R}=0.012 \\
C_{I}=0.0066
\end{array}\right.
$$

The system of governing equations, Eqs. (1-3), is closed by making assumptions on the thermodynamics of the gas considered. It is assumed that the gas is thermally perfect, i.e. it obeys the gas law. Furthermore, the gas is assumed to be calorically perfect, which implies that internal energy and enthalpy are linear functions of temperature. 


\section{Sound Propagation}

Sir James Lighthill's description of his acoustic analogy, ${ }^{12}$ i.e. the analogy between the full nonlinear flow and the linear theory of acoustics, is often referred to as the start of aeroacoustics. Combining the time derivative of the continuity equation and the divergence of the momentum equation, the flow equations may be rewritten in a wave operator form. ${ }^{12}$ An analytical solution may be obtained by the use of a free-space Green's function and thus acoustic pressure signals in far-field observer locations can be estimated by integration over a volume containing all sound-generating sources. It is important to note that no interaction between the propagating sound wave and the flowfield in which it is propagating is explicitly considered when the integral form of the analogy is used, i.e. no refraction or convection effects are taken into account. The equation produced by this pioneer work has later been modified in several ways to include, for example, the effects of the mean-flow acoustic interactions, flow inhomogeneities and surfaces. ${ }^{21}$

\section{A. Kirchhoff Surface Integral Formulation}

Kirchhoff integration is a method for predicting the value of a property, $\Phi$, governed by the wave equation, at a point outside a surface enclosing all generating structures. ${ }^{22}$ The method was originally used in the theory of diffraction of light and in other problems of an electromagnetic nature ${ }^{1}$ but has recently been extensively used for aeroacoustic applications. The integral relation is given by

$$
\Phi(\mathbf{y}, t)=\frac{1}{4 \pi} \int_{S}\left[\frac{\Phi}{r^{2}} \frac{\partial r}{\partial n}-\frac{1}{r} \frac{\partial \Phi}{\partial n}+\frac{1}{c_{\infty} r} \frac{\partial r}{\partial n} \frac{\partial \Phi}{\partial t}\right]_{\tau_{r}} d S(\mathbf{x})
$$

where $\mathbf{y}$ is a observer location in the far field and $\mathbf{x}$ a location on the surface. $\tau_{r}$ denotes that the expression within brackets is to be evaluated at a retarded time, i.e. emission time. $\tau_{r}$ is related to the observer evaluation time, $t$, by the distance from the surface to the observer, $r=|\mathbf{y}-\mathbf{x}|$, and the speed of sound in the far-field region, $c_{\infty}$, as

$$
\tau_{r}=t-\frac{r}{c_{\infty}}
$$

The variable $\Phi$ that is to be evaluated in this case is the surface pressure. $S$ denotes the surface enclosing all sound generating structures and $n$ denotes the direction normal to the surface. Surface $S$ must be placed in a region where the flow is completely governed by a homogeneous linear wave equation with constant coefficients. ${ }^{3}$ Greater detail on the Kirchhoff surface integration method is given in e.g. Freund et al. ${ }^{3}$ and Lyrintzis. ${ }^{22}$

The strength of the sources in the hydrodynamic jet region decays slowly downstream, which means that the downstream end of a closed surface will be located in regions of considerable hydrodynamic fluctuations. It is thus common practice to use Kirchhoff surfaces that are not closed in the upstream and downstream ends. Freund et al. ${ }^{3}$ showed that the errors introduced by using such surfaces are small if the main portion of the sound sources are within the axial extent of the surface and if lines connecting observer locations with locations in the hydrodynamic region, representing the main sources of sound, intersect the surface. Rahier et al. ${ }^{23}$ found that the downstream closing surface makes only a minor contribution to the radiated sound. In the work reported in this paper a Kirchhoff surface open in both the upstream end and the downstream end is used, see Figure (2).

The Kirchhoff surface integral method is less computationally expensive and storage demanding than a method based on volume integrals, e.g. Lighthill's acoustic analogy, since it is only surface data that must be stored and evaluated. Moreover, the integrand includes only first-order derivatives, while the integrand in Lighthill's acoustic analogy contains second-order derivatives in time or space. One of the main strengths of the Kirchhoff surface integral approach, contrary to many other methods, is that as long as all sources of sound, flow inhomogeneities and objects that in some way affect the radiated sound are bounded by the surface, all these effects are taken into account. This means, for 


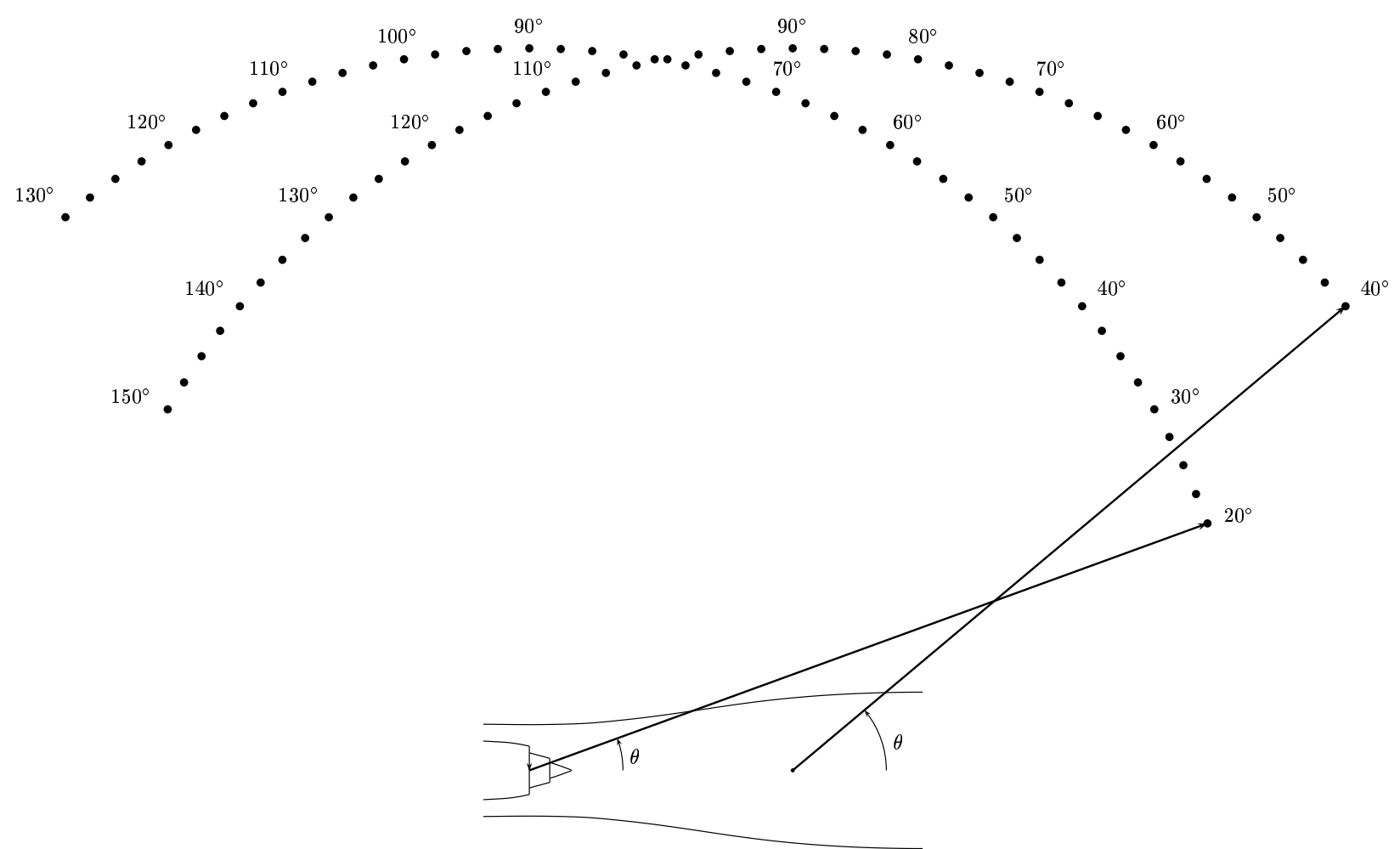

Figure 2. Kirchhoff integration surface and far-field observer locations are depicted. In total 90 observer locations are represented; 45 observers are situated on an arc centered on the centerline at the exit plane of the secondary nozzle and 45 observers on an arc centered at a location $1.5 \mathrm{~m}$ downstream of the first location. The latter location is approximately where the potential core ends in RANS computations made for the same case.

example, that no extra treatment is needed to be able to predict the effects of refraction and convection. This is one of the attractive features of this method. Moreover, both a strength and a weakness of the surface integral approach is the effect of numerical dissipation on the predicted sound. Acoustic waves approaching the integration surface will be low-pass filtered by the numerical method. A result of this artificial dissipation is that spurious high-frequency noise not supported by the mesh and scheme will be filtered out and thus not contribute to the predicted far-field sound. On the other hand, the numerical dissipation affects the ability to capture the high-frequency range of the acoustic pressure spectra, and thus the quality of the prediction depends strongly on the mesh resolution in the Kirchhoff surface region. The ideal situation would be to place the integration surface as far as possible from the sources to ensure that all non-linearities appear within the integration surface. However, the distance from the source to the surface is often limited by the numerical dissipation introduced by the method. Using Ffowcs William \& Hawkings ${ }^{2}$ surface integral method on a porous surface formulation allows the surface to enter regions with weak hydrodynamic fluctuations, which means that the surface can be placed somewhat closer to the sources of sound, see e.g. Shur et al. ${ }^{18}$ Rahier et al. ${ }^{23}$ and Uzun et al. ${ }^{4,5}$ However, if placed in the linear acoustic field the Kirchhoff surface formulation and the formulation of Ffowcs William \& Hawkings gives identical results. Uzun et al. ${ }^{4,5}$ found the results obtained using the two surface integration methods to be similar for integration surfaces placed rather close to the jet. 


\section{Method}

\section{A. Numerical Scheme}

The Favre-filtered Navier-Stokes equations were solved using a finite-volume method solver with a third-order low-dissipation upwind scheme for the convective fluxes and a centered difference approach for the diffusive fluxes. The temporal derivatives were estimated using a second-order three-stage Runge-Kutta technique. The solver used is based on the G3D family of codes developed by Eriksson. ${ }^{24}$ The convective scheme is a combination of centered and upwind biased components that have been used with good results for LES of free shear flows by Mårtenson et al. ${ }^{25}$ and, more recently, for shock/shear-layer interaction by Wollblad et al. ${ }^{26}$ The coefficients of low-dissipation upwind scheme for estimation of the convective flux over a cell face are derived using a third-order polynomial $Q(x)$ to represent the variation of the flow state in the direction normal to the face as

$$
Q(x)=A+B x+C x^{2}+D x^{3}
$$
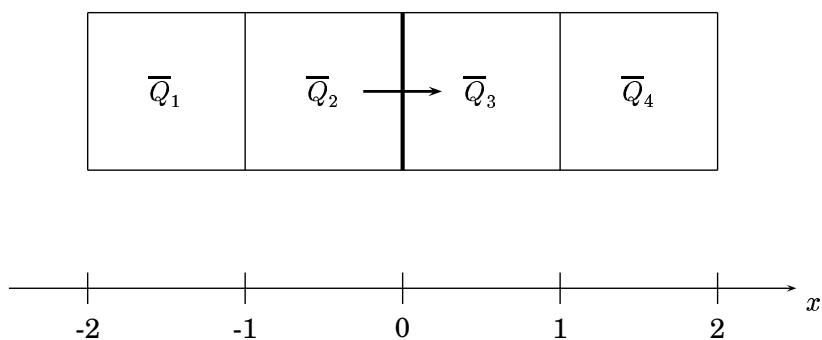

Figure 3. The face state, $Q_{0}$, is estimated using the state in four neighboring cells.

The face state is evaluated using the interpolated value $Q(0)$, modified to include upwinding by adding the third derivative of $Q(x)$ according to

$$
\begin{aligned}
Q_{0} & =Q(0)+\delta Q^{\prime \prime \prime}(0)=A+6 \delta D= \\
& =C_{1} \bar{Q}_{1}+C_{2} \bar{Q}_{2}+C_{3} \bar{Q}_{3}+C_{4} \bar{Q}_{4}
\end{aligned}
$$

where coefficient $\delta$ in front of the upwind term has been chosen by numerical experiments ${ }^{25}$ to be $(1 / 96)$ in order to introduce only a small amount of upwinding. The result is the low-dissipation third-order upwind scheme with the coefficients

$$
\left\{\begin{array}{l}
C_{1}=-\left(\frac{1}{12}+\delta\right)=-\frac{9}{96} \\
C_{2}=\left(\frac{7}{12}+3 \delta\right)=\frac{59}{96} \\
C_{3}=\left(\frac{7}{12}-3 \delta\right)=\frac{53}{96} \\
C_{4}=-\left(\frac{1}{12}-\delta\right)=-\frac{7}{96}
\end{array}\right.
$$

For more detail on the numerical scheme, see Eriksson ${ }^{24}$ or Andersson. ${ }^{8}$

\section{B. Computational Set-up}

The computational domain is divided into three parts: a high-resolution LES region near the nozzle walls, a medium dense LES region and a coarse LES region. Figure (4) shows the location of these three mesh regions. The maximum size of the computational cells in the medium-resolution region is 
chosen such that the mesh in this region will support acoustic waves of frequencies up to $15 \mathrm{kHz}$, using 8 cells per wavelength. In this region the cells are approximately equidistant. In the high-resolution LES region, the cells are twice as fine in each direction as in the medium dense region and the cells are stretched somewhat towards the nozzle walls. The cells in the coarse LES region are twice as wide in each direction as those in the medium-resolution LES region. The computational domain was discretized using a block-structured boundary-fitted mesh with 159 mesh blocks and approximately $2.0 \times 10^{7}$ nodes. Figure (5) depicts the mesh in the near wall fine LES region. The mesh is constructed using a combination of Cartesian and polar mesh blocks so as to ensure mesh homogeneity in the radial direction throughout the domain. A block with square cross section is used at the centerline to avoid a centerline singularity.

In order to minimize the effect of reflections at the domain outlet on the predicted flowfield, a damping zone was added at the domain outlet. The functionality of this damping zone, or buffer layer, is described in more detail in Andersson et al. ${ }^{6}$ and Andersson. ${ }^{7}$ At the nozzle inlet total pressure and total enthalpy are specified. Entrainment velocities at the coarse LES domain boundary are obtained by using a 2D extension of the domain representing the flow outside the LES domain, see Figure (4). This procedure will be discussed in more detail in Section D.

Although the flow is rather well resolved in the near wall regions, since the Reynolds number is very high, this must be considered as being a coarse LES. To be able to represent the near wall behavior of the flow wall functions are used where needed.

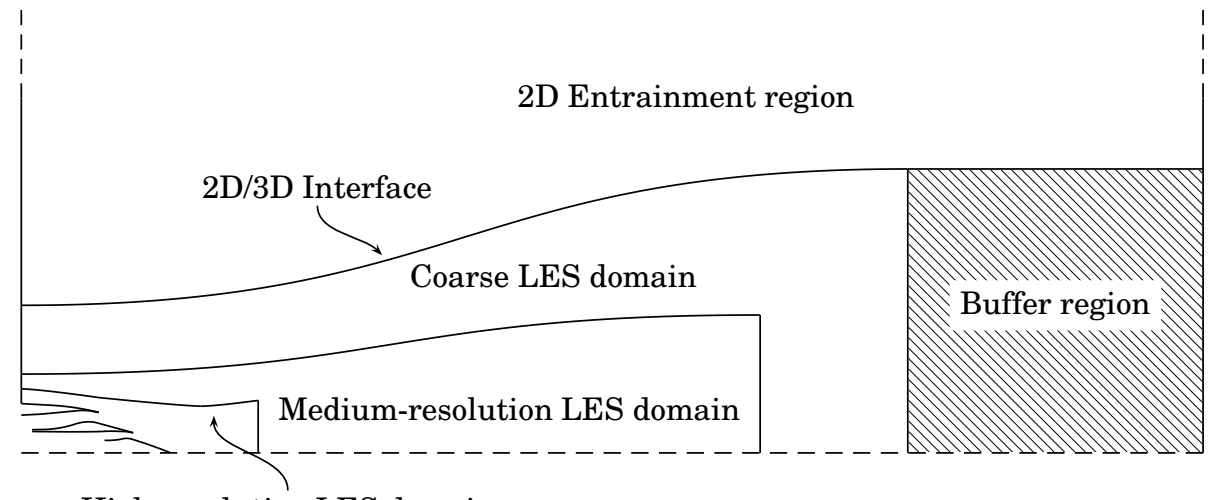

High-resolution LES domain

$0.8[\mathrm{~m}] \longrightarrow 2.2[\mathrm{~m}] \mathrm{\longleftrightarrow} \longleftrightarrow{ }_{0.5[\mathrm{~m}]}^{\longleftrightarrow} \longleftrightarrow 1.0[\mathrm{~m}]$

Figure 4. The computational domain is divided into three regions: a high-resolution region for resolution of boundary layers and initial shear layers, a medium LES region optimized for propagation of acoustic waves and a coarse LES region.

\section{Stepwise Increased Resolution}

To keep the numerical accuracy high and thus minimize the low pass filtering of the acoustic waves as they travel through the calculation domain, the mesh has to be rather fine even in the far-field regions. 


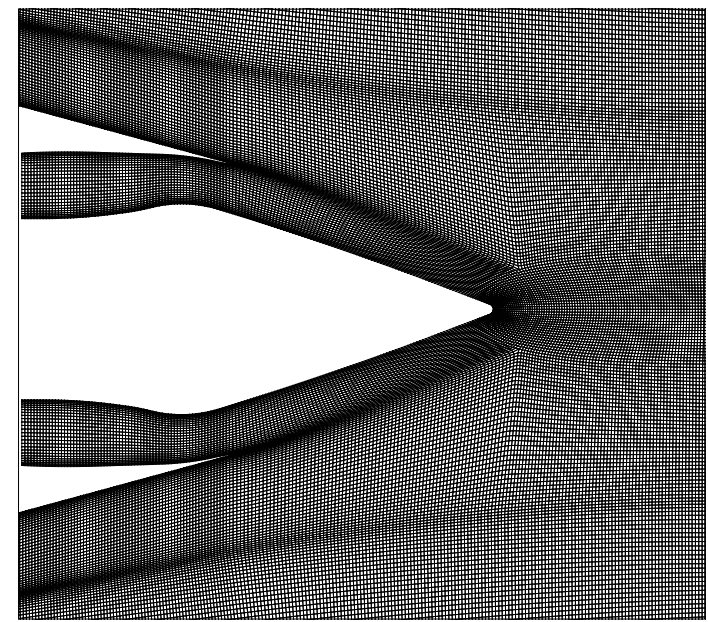

(a) A slice through the calculation domain made at $z=0$, i.e. a $x y$-plane, in the fine LES region is depicted. The figure shows the primary flow nozzle and the center body.

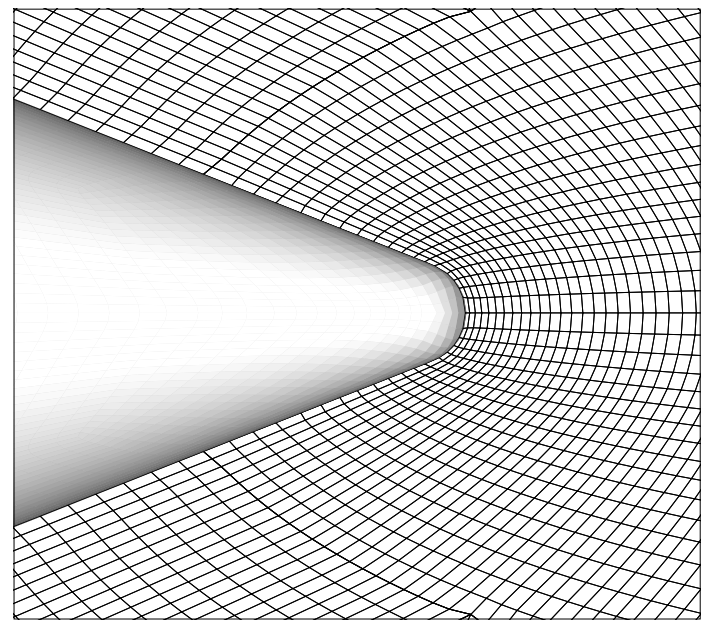

(b) A close up of the cone tip region is depicted. The mesh in this region is generated by mapping the $x$-plane surface of a square Cartesian block starting at the cone tip to the cone surface.

Figure 5. Mesh slices in the fine LES near-nozzle region

Moreover, it is desirable to have a mesh as equidistant as possible in order to minimize the introduction of numerical errors. The high resolution in the far field regions together with the extreme demands for resolution near the nozzle walls and in the shear layers results in that a very large number of cells have to be used to discretize the computational domain. To keep the number of cells down and at the same time being able to ensure high resolution near the nozzle walls and in the initial shear layer regions, the mesh used in this work has been refined in two distinct steps. In each of these steps the number of cells is increased by a factor two in each direction. In order to minimize the introduction of numerical errors, special treatment is utilized for estimation of the convective and diffusive fluxes over the interfaces for which this kind of refinement occurs, see Figure (6). The flux over the cell face one step in on the coarse side of the interface, i.e. at $x=-1$, is obtained using a corresponding coarse cell on the fine side, constructed as the average of the closest eight fine cells, see Figure (6b). For the block interface and the face one step in on the fine side $(x=0.5)$, cell reconstruction is used to generate corresponding fine cells on the coarse side of the interface, see Figure (6c). The fluxes over the interface are evaluated on the fine side and corresponding flux contributions with opposite signs are added to the cell on the coarse side.

In the cell reconstruction procedure it is assumed that the spatial variation of the state vector in one direction can be represented by a second-order polynomial, $Q(x)=A+B x+C x^{2}$. By integration of this polynomial over three cells the coefficients $A, B$ and $C$, can be expressed as functions of the cell averages of the state vector according to

$$
\begin{array}{lll}
A= & \frac{1}{24} & {\left[-\bar{Q}_{1}+26 \bar{Q}_{2}-\bar{Q}_{3}\right]} \\
B= & \frac{1}{2} & {\left[-\bar{Q}_{1}+\bar{Q}_{3}\right]} \\
C= & \frac{1}{2} & {\left[\bar{Q}_{1}-2 \bar{Q}_{2}+\bar{Q}_{3}\right]}
\end{array}
$$




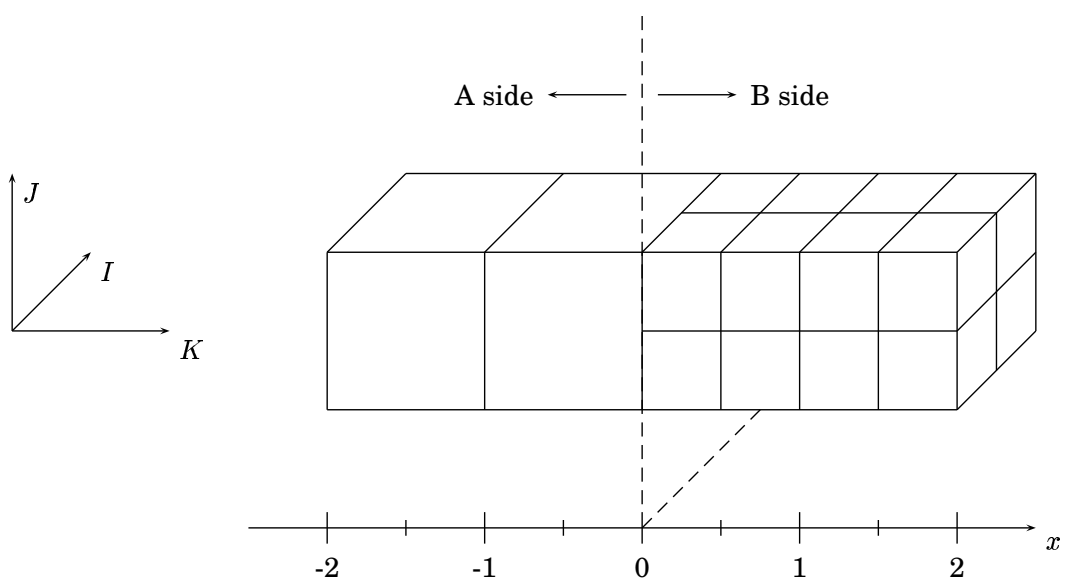

(a)

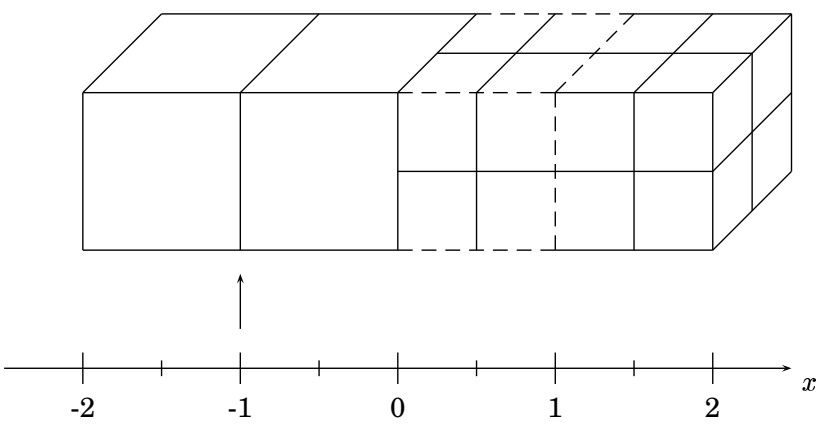

(b)

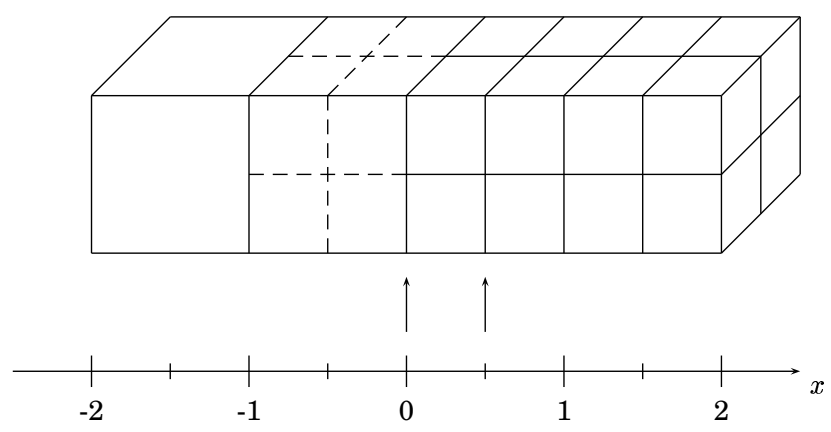

(c)

Figure 6. Over the interface between mesh block $A$ and mesh block $B$, the number of cells is increased by a factor two in each direction (a). The fluxes one step in on the A side of the interface, i.e. at $x=-1$, are estimated by using a corresponding coarse cell on the fine side obtained by averaging over eight fine cells (b). The fluxes on the block interface and one step in on the $B$ side, i.e. at $x=0.5$, is estimated using cell reconstruction on the coarse side of the interface $(c)$.

The cell average of the state vector for a reconstructed cell is calculated by integration according to

$$
\bar{Q}=\frac{1}{x_{2}-x_{1}} \int_{x_{1}}^{x_{2}} Q(x) d x
$$

where $x_{1}$ and $x_{2}$ represents the boundary locations of the new cell. The cell reconstruction is made in two steps. In a first step, both of the cells on the coarse side are divided in the $I$ and $J$ directions into four cells, matching those on the fine side of the interface. In this operation only the neighboring cells on the coarse side of the interface are used, see Figure (7). As an example of the reconstruction procedure, the state vector for the cell in the upper right corner in Figure (7) is obtained as

$$
\begin{aligned}
\bar{Q}_{i, j, k}^{++}= & \frac{1}{64}\left[-\bar{Q}_{i-1, j+1, k}+\bar{Q}_{i-1, j-1, k}+\bar{Q}_{i+1, j+1, k}-\bar{Q}_{i+1, j-1, k}\right]+ \\
& +\frac{1}{8}\left[-\bar{Q}_{i-1, j, k}+\bar{Q}_{i+1, j, k}-\bar{Q}_{i, j-1, k}+\bar{Q}_{i, j+1, k}\right]+\bar{Q}_{i, j, k}
\end{aligned}
$$

The new cells closest to the interface on the coarse side are now divided in the direction normal to the interface by cell reconstruction involving two layers of cells on each side of the interface. In this 

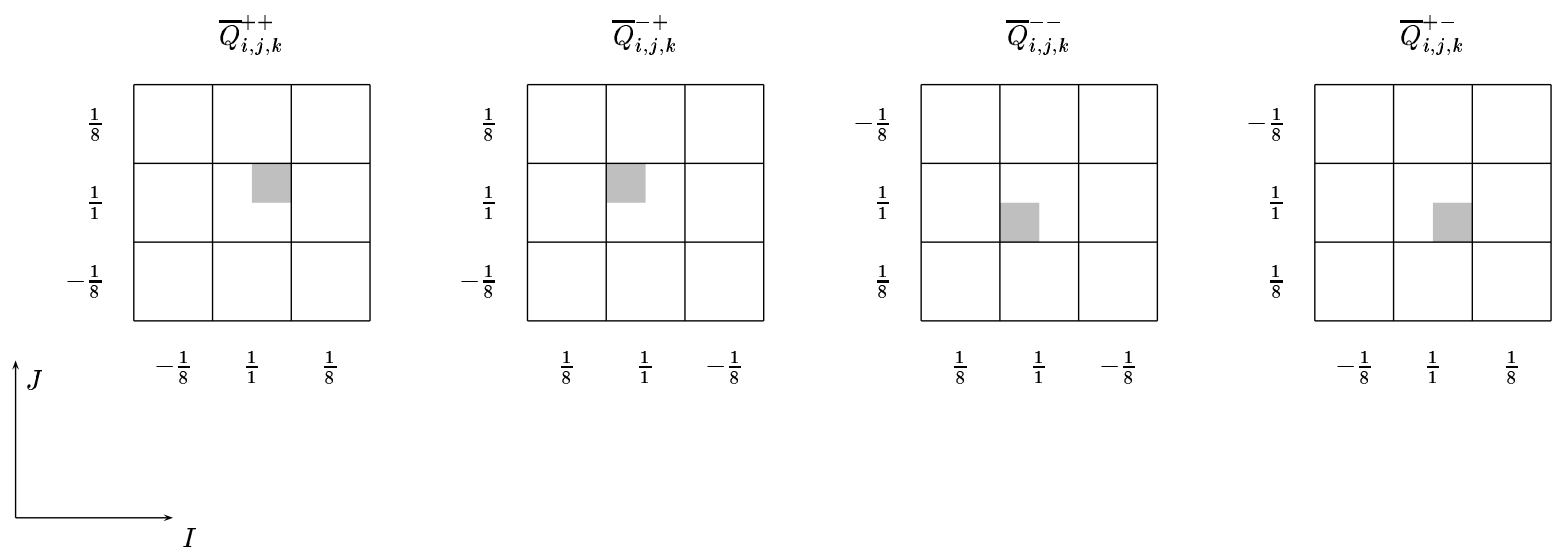

Figure 7. Cell reconstruction (step 1). Generate cells on the coarse side of the interface, matching the cells on the fine side. This step only involves cells on the coarse side.

reconstruction step, the average of the two B-side cells is used to represent the fine side of the interface, see Figure (8).

$$
\bar{Q}_{i, j, k}^{+++}, \bar{Q}_{i, j, k}^{-++}, \bar{Q}_{i, j, k}^{-+}, \bar{Q}_{i, j, k}^{+-+}
$$

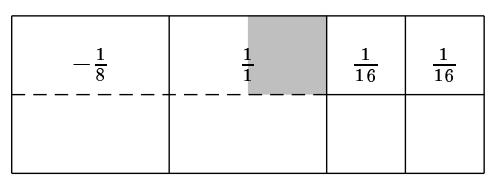

$$
\bar{Q}_{i, j, k}^{++-}, \bar{Q}_{i, j, k}^{-+-}, \bar{Q}_{i, j, k}^{---}, \bar{Q}_{i, j, k}^{+--}
$$

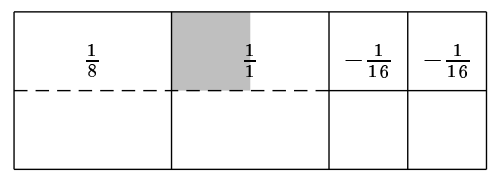

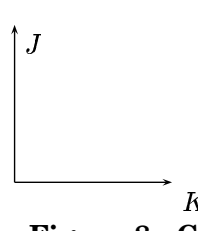

Figure 8. Cell reconstruction (step 2). The cells on the coarse side are reconstructed to match those on the fine side of the interface. In the reconstruction the average of two cells on the fine side are used to represent the fine side of the interface.

\section{Entrainment Boundary Conditions}

The radial extent of the computational domain increases downstream such that the flow in the domain boundary region can be assumed to be irrotational and axisymmetric. Hence, the flow outside the three-dimensional computational domain can be represented by a less expensive two-dimensional axisymmetric calculation, see Figure (4). The domain for this $2 \mathrm{D}$ calculation extends 4.5 meters radially from the jet centerline. To minimize boundary reflections, the 2D/3D interface acts as an absorbing boundary condition on both sides. The flow properties of the boundary conditions for the 3D domain are specified using flow information from the 2D domain, and the boundary condition for the 2D domain is defined using azimuthally averaged flow information from the $3 \mathrm{D}$ domain, see figure (9). The 2D domain has an extent in the tangential direction corresponding to the width of the scheme used for the convective fluxes, i.e. four cells. Azimuthal averages are obtained for the four cell rows adjacent to the interface highlighted in figure (9). Rotational periodic interfaces are used for the block faces in the tangential direction for the 2D domain. This entrainment boundary approach enables the use of a 
rather narrow 3D domain. Furthermore, the outer boundaries of the 2D domain are located relatively far from the jet axis, which simplifies the definition of these boundary conditions.

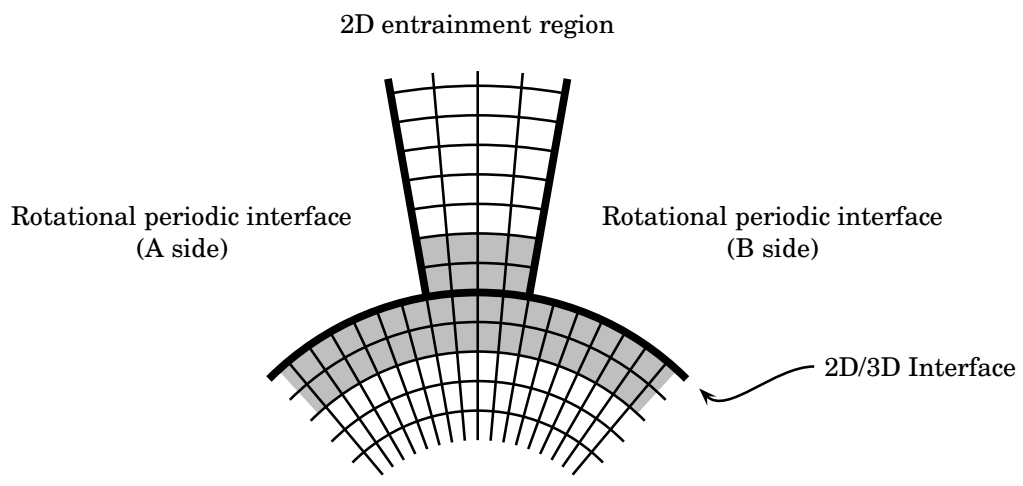

3D LES domain

Figure 9. Interface between the 2D entrainment region and the 3D region. The interface acts as an absorbing boundary condition on both sides. The flow information specified at the interface boundary is obtained using azimuthally averaged flow information on both sides of the interface. The filled area denotes cells used to obtain these azimuthally averaged flow quantities.

\section{Results}

In this section results for two simulations made for the coaxial nozzle configuration will be presented. These two simulations will hereafter be referred to as case 1 and case 2, respectively. Case 1 is the result of a first preliminary large-eddy simulation of the coaxial jet configuration. After evaluation of case 1, three changes were made to the simulation set-up for reasons that will be discussed in more detail later. The second case was simulated with these changes included and the effects on the predicted flowfield will be discussed in some detail in this section. The changes made were the following:

1. The SGS filter width was decreased from $\Delta=\left(\Delta_{1} \Delta_{2} \Delta_{3}\right)^{(1 / 3)}$ in case 1 to $\Delta=\min \left(\Delta_{1}, \Delta_{2}, \Delta_{3}\right)$ in case 2 .

2. When case 1 was run, it was found that selective artificial damping for density was needed to ensure stability in the simulation. In case 2 this artificial damping was replaced with a TVD limiter for density. This results in a less dissipative scheme and thus improves the numerical accuracy. As will be shown later this change had some positive effects on the shear layer flow.

3. In case 1 , constant values of $P_{0}$ were specified at the nozzle inlet boundaries for both the core flow and the bypass flow whereas for case 2 the inlet total pressures were given as profiles, $P_{0}=P_{0}(r)$, which increased the boundary layer thickness in this simulation.

In the first simulation it was found that the mixing in the outer shear layer was very low close to the nozzle. Once the transition process was initiated, large ring shaped structures were generated, which eventually broke down into smaller structures. However, the low degree of mixing in the initial shear layer region results in that the ring shape of the vortex structures keeps their identity quite far downstream of the nozzle exit. This led to levels of turbulence kinetic energy significantly higher than what was obtained in RANS computations for the same nozzle configuration and flow conditions. 
Although the lower levels of turbulence kinetic energy found in the RANS computations are not considered as a proof of that the LES results are unphysical, it gives an indication of that something might be wrong. It was found that the shear layer between the core flow and the bypass flow was very thin and stable. This was, unlike the previously mentioned observation, seen in the RANS results as well, which gives an indication of that this flow behavior might be correct. Nevertheless, this was the reason for changing the nozzle inlet boundary conditions and improve the accuracy of the numerical scheme by replacing the artificial damping with a TVD limiter. A velocity profile ought to give thicker boundary layers and hence a thicker shear layer. The observations for the case 1 jet mentioned in this paragraph led to the changes of the simulation set-up listed above.

Although the initial jet region is well resolved, the aspect ratio of the cells in the nozzle regions is high. The cells are quite wide in the tangential direction as compared to the radial direction. Using $\Delta=$ $\left(\Delta_{1} \Delta_{2} \Delta_{3}\right)^{(1 / 3)}$ as the filter width in the Smagorinsky subgrid-scale model leads to that the amount of dissipation introduced is not representative for the resolution in the radial direction. Several possible representations of the filter width for cells with high aspect ratio are described by Sagaut. ${ }^{27}$ However, in this case the shortest side of each cell was chosen as a rough estimate. Decreasing the filter width to $\Delta=\min \left(\Delta_{1}, \Delta_{2}, \Delta_{3}\right)$ enhanced the mixing in the outer shear layer. The change from artificial damping to a TVD limiter further increased the degree of mixing in the outer shear layer. Moreover, this change led to a more unstable inner shear layer. A probably more physically correct behavior of the outer shear layer was established in the second simulation.

The time-average results presented in Section B and the two-point space-time correlations presented in Section $\mathrm{C}$ have been obtained from databases of samples for a subset of the computational domain using a sample rate of $100[k H z]$. 650 samples were obtained for case 1 and 1500 samples for case 2 . The time step used was chosen such that the CFL number did not exceed 0.5, which gave a time step of $\Delta t=1.0 \times 10^{7}[\mathrm{~s}]$, i. e. a sample rate of $100[\mathrm{kHz}]$ means that data are saved every hundred time step.

\section{A. Instantaneous Flowfield}

This section gives some examples of snap shots of the flow for case 1 and case 2 . Figures (10) and (11) show contours of density for case 1 and case 2, respectively. From these figures, the differences in the two simulations regarding the initial mixing are quite visible. The initial part of the outer shear layer in the case 1 simulation has no structures present at all whereas in case 2 , small vortical structures are generated directly at the nozzle lip. As discussed previously, the lack of initial mixing in case 1 leads to that the shape of vortical structures generated in the initial shear layer remain quite far downstream of the nozzle exit, which means that these structures grow quite large in size before they are broken down into smaller structures. The degree of mixing in the shear layer between the core flow and the bypass flow is generally very low. This is especially noticeable in the first simulation where this shear layer becomes more or less a surface separating the two streams. The vortical structures in the outer shear layer affect this surface in that the pairing processes occurring in the outer shear layer generate a wave-like behavior of the surface, see Figure (10). This finally leads to the collapse and break up of the shear layer/surface near the end of the inner potential core region. The inner shear layer seems to be rather stable in case 2 as well but in this case small structures are generated in the initial part of the shear layer. The reason for the low degree of mixing in the inner shear layer is believed to be physically correct and caused by a combination of stabilizing factors. First of all, the velocity and momentum ratios are reversed, i.e. the velocity in the primary stream is higher than the velocity in the secondary stream, but, owing to temperature differences, the momentum flux is lower in the primary stream than in the secondary stream. This might have a stabilizing effect on the shear layer between the two streams. Moreover, the flow in the initial part of the inner shear layer is not fully axial but has a negative velocity component in the radial direction, which makes the shear layer concave. This curvature of the shear layer might also have a stabilizing effect on the flow. Comparing Figure (10)

15 of 26 
with Figure (11), the structures generated once the inner shear layer breaks up is significantly larger in case 1 than in case 2 . This might be an effect of the large coherent structures that are present in the outer shear layer in the case 1 jet.

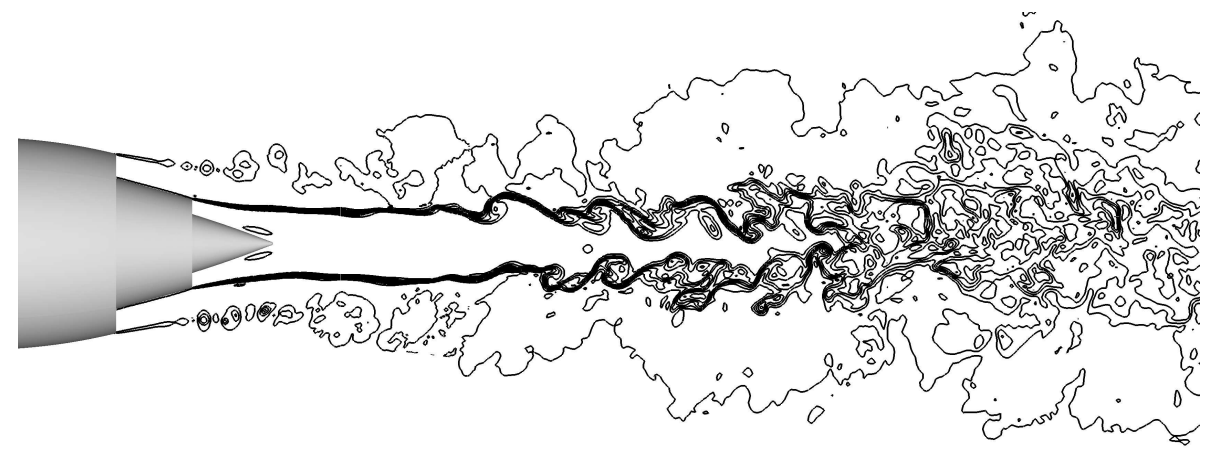

Figure 10. Instantaneous density contours (case 1)

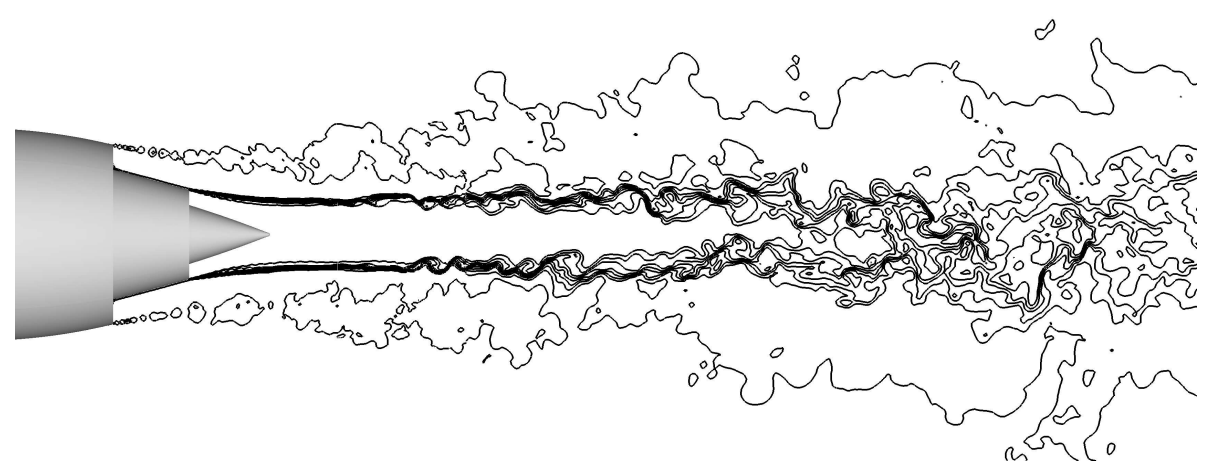

Figure 11. Instantaneous density contours (case 2)

Figures (12) and (13) show instantaneous contours of entropy, $s=P / \rho^{\gamma}$. Again the differences in initial mixing for the two jets are quite visible. Comparing the figures, it seems that the potential core is longer in the latter case, which might be explained by the fact that the mixing in case 1, once initiated, is more violent and efficiently breaks the potential core region down.

Figures (14(a)) and (14(b)) again show contours of entropy in the initial shear layer region. The thicker shear layers seen in case 2 is probably an effect of that the inlet total pressures have been specified as profiles in this case.

In Figures (15-16) iso surfaces of density visualizing the flow structures in the inner and outer shear layers are shown. As can be seen in Figure (15), although the shear layers seems to be stable in both cases, the structures that occurs in the iso surfaces obtained for case 1 are very different from those in the iso surfaces obtained for case 2 . In case 1 only large structures can be seen as was indicated in Figure (10). These large structures in the inner shear layer arise when vortical structures in the outer shear layer affect the inner shear layer. It is these large structures that eventually lead to the break-up of the inner shear layer in case 1. In case 2, Helmholtz instabilities in the form of ring shaped vortical structures can be seen in the initial part of the inner shear layer. As the shear layer develops, these vorticies are deformed and axial structures are generated. This leads to that the inner shear 


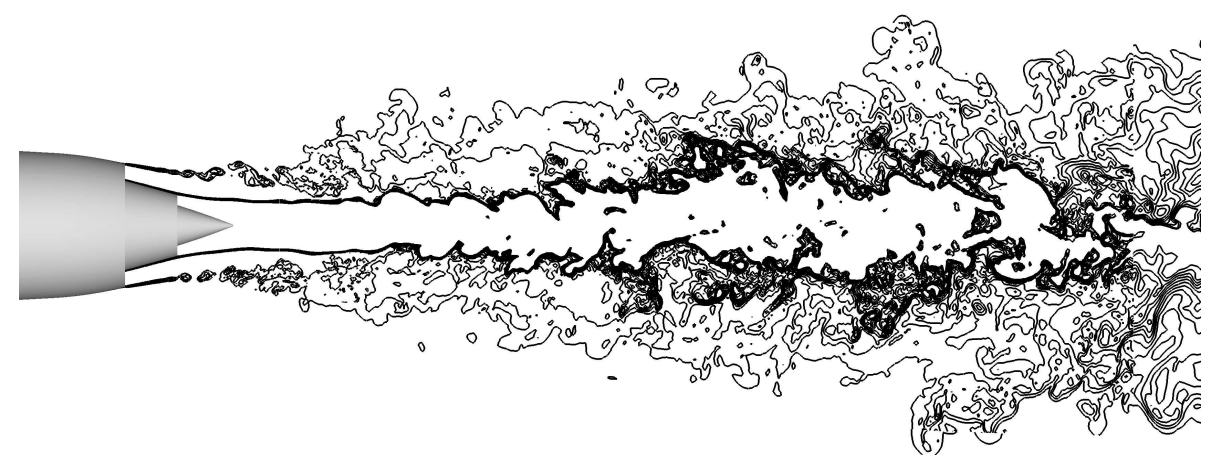

Figure 12. Instantaneous entropy contours (case 1)

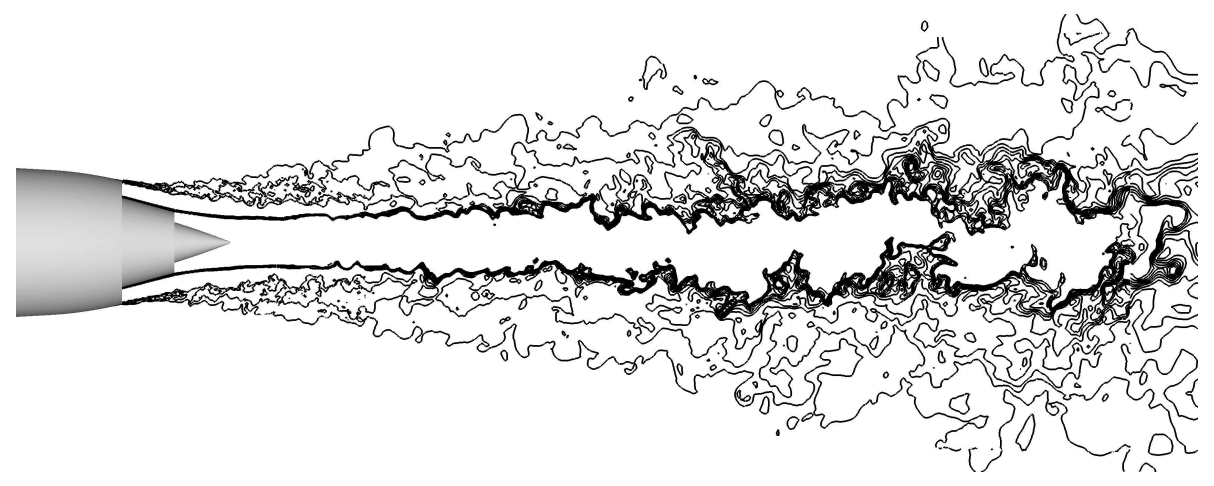

Figure 13. Instantaneous entropy contours (case 2)

17 of 26

American Institute of Aeronautics and Astronautics Paper 2005-2884 


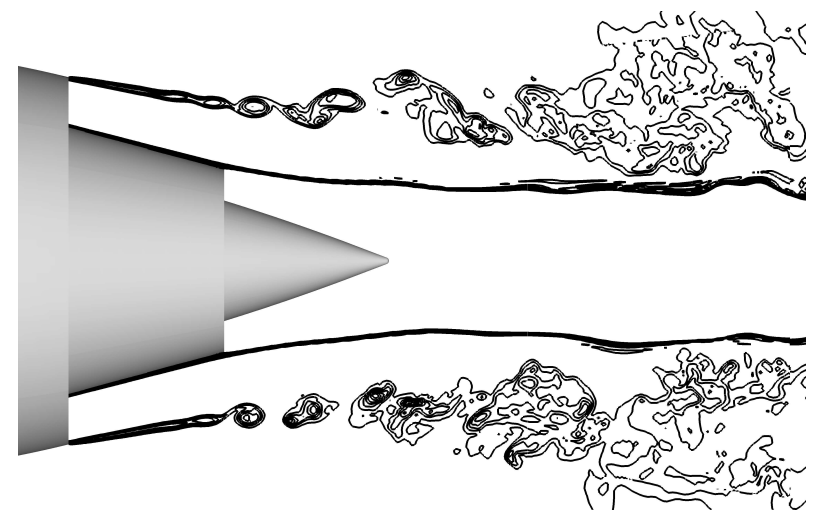

(a) case 1

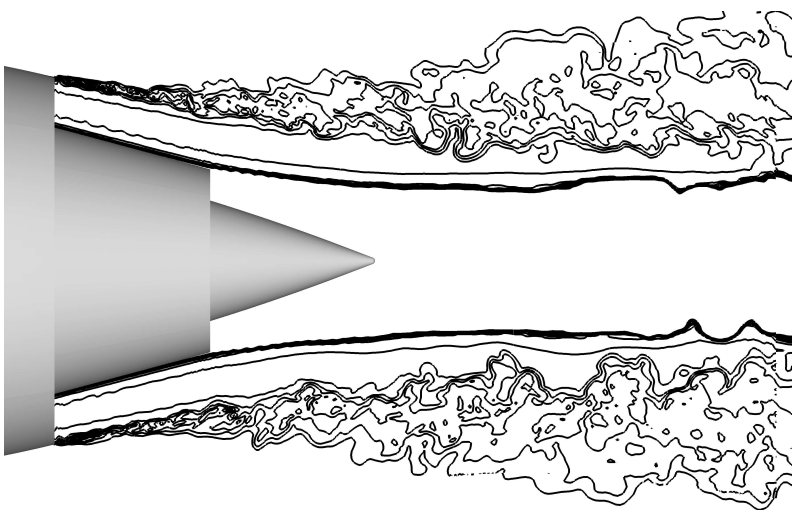

(b) case 2

Figure 14. Instantaneous contours of entropy near the nozzle walls.

layer breaks up at an earlier stage in case 2 than in case 1. Figures (16(a)) and (16(b)) again shows the differences in the initial part of the outer shear layer in the two cases. The initial mixing process is enhanced in case 2 , which leads to that smaller flow structures are generated close to the nozzle lip than in case 1.

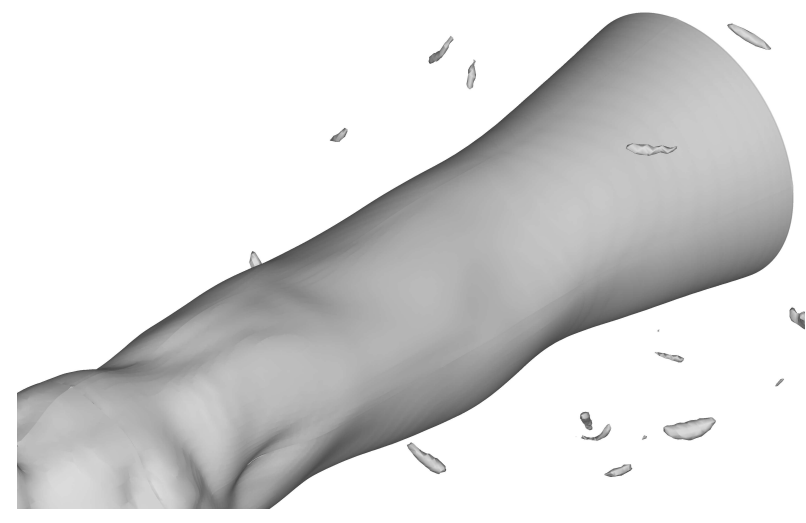

(a) case 1

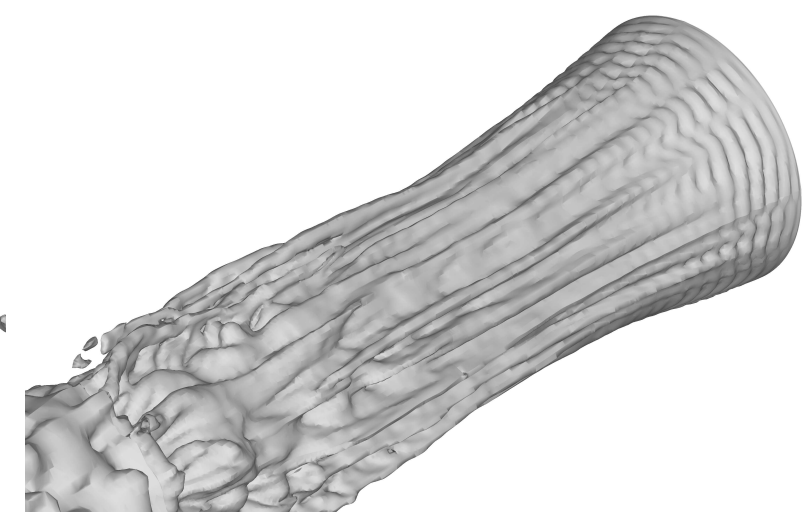

(b) case 2

Figure 15. Density iso surfaces showing flow structures in the shear layer between the primary and secondary stream.

\section{B. Time-averaged Flowfield}

This section presents profiles of time-averaged flow quantities. Data have been obtained along three axial lines and ten radial lines in the near-nozzle region. The axial lines are situated at the centerline, $r=0$, and along the nozzle lip lines, i.e. $r=D_{p} / 2$ and $r=D_{s} / 2$. The axial location of the first radial profile is $x=0.018 D_{s}$ and the distance between each radial profile is $\Delta x=0.043 D_{s}$. All profiles 


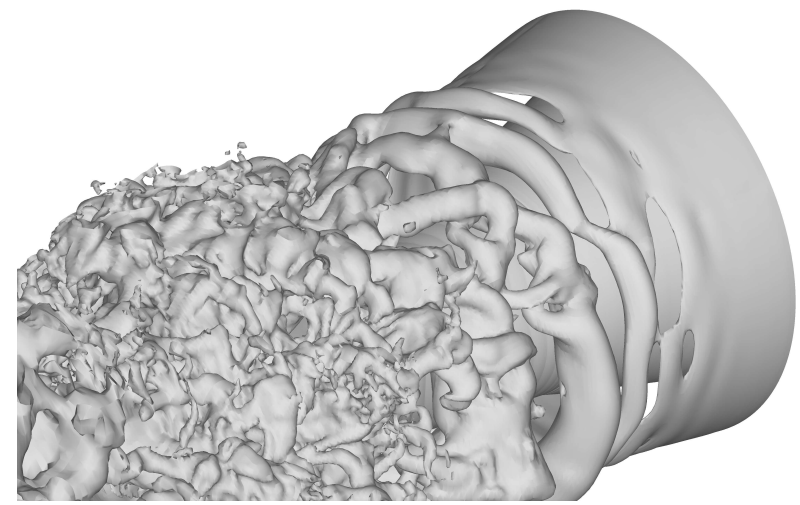

(a) case 1

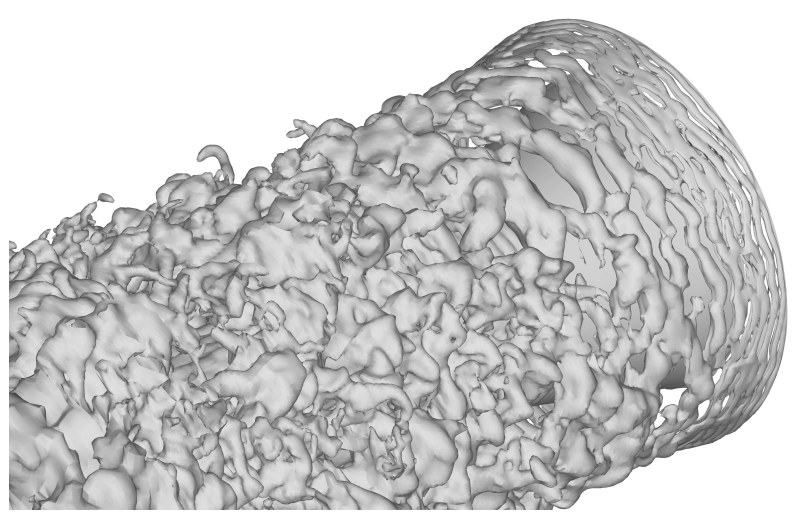

(b) case 2

Figure 16. Density iso surfaces showing flow structures in the shear layer between the secondary stream and the stagnant surrounding fluid.

except the one extracted along the centerline have been obtained using data averaged in both time and in the azimuthal direction so as to improve the statistical convergence. It should be noted that the axial extent of the subset of the computational domain for which the databases of samples for the two simulations have been generated does not reach the as far downstream of the nozzle exit as to the end of the potential core. Therefore, no information on the predicted potential core lengths can be extracted from the time-averaged data.

Figure (17) shows axial and radial profiles of axial velocity. As can be seen from Figure (17(a)), there are some non-negligible differences in the development of axial velocity in the shear layer regions and along centerline in the two simulations. For example, case 1 shows a longer low velocity region along the centerline downstream of the center body. For the radial profiles, however, only small deviations can be identified, see Figure (17(b)). For the radial profiles obtained close to the nozzle, the effects of the two shear layers are clearly visible. For the profile furthest downstream these effects are almost gone and the profile looks more like a single-stream jet profile. Figure (18) shows profiles of $u_{r m s}$. In these profiles there are significant differences between the two cases. The turbulence intensity is higher close to the nozzle in case 2 than in the case 1 simulation in which $u_{r m s}$ is almost zero at the nozzle lips, see Figure (18(a)). At locations further downstream the intensities are higher in case 1 than in case 2 . The same trend can be seen in Figures (19-20), which show resolved turbulence intensity and $u^{\prime} v^{\prime}$-correlation, respectively. These results are consistent with the trends seen in the instantaneous flowfield images presented in the previous section, i.e. the jet obtained in the case 2 simulation starts to mix immediately as the jet exits the nozzle whereas the shear layers in case 1 develops slower. However, once the mixing is initiated the mixing process is more violent in case 1 and thus the resolved fluctuations reach higher levels.

\section{Two-point Space-time Correlations}

Two-point space-time correlations were obtained for the axial velocity component in a few shear layer locations along the nozzle lip line of the outer nozzle. The two-point space-time correlation of axial velocity for a certain spatial separation, $\xi$, and separation in time, $\tau$, is given by

$$
\mathcal{R}_{u u}(\mathbf{x}, \xi, \tau)=\frac{\left\langle u^{\prime}(\mathbf{x}, t) u^{\prime}(\mathbf{x}+\xi, t+\tau)\right\rangle_{t \theta}}{\sqrt{\left\langle u^{\prime 2}(\mathbf{x})\right\rangle_{t \theta}} \sqrt{\left\langle u^{\prime 2}(\mathbf{x}+\xi)\right\rangle_{t \theta}}}
$$

19 of 26 


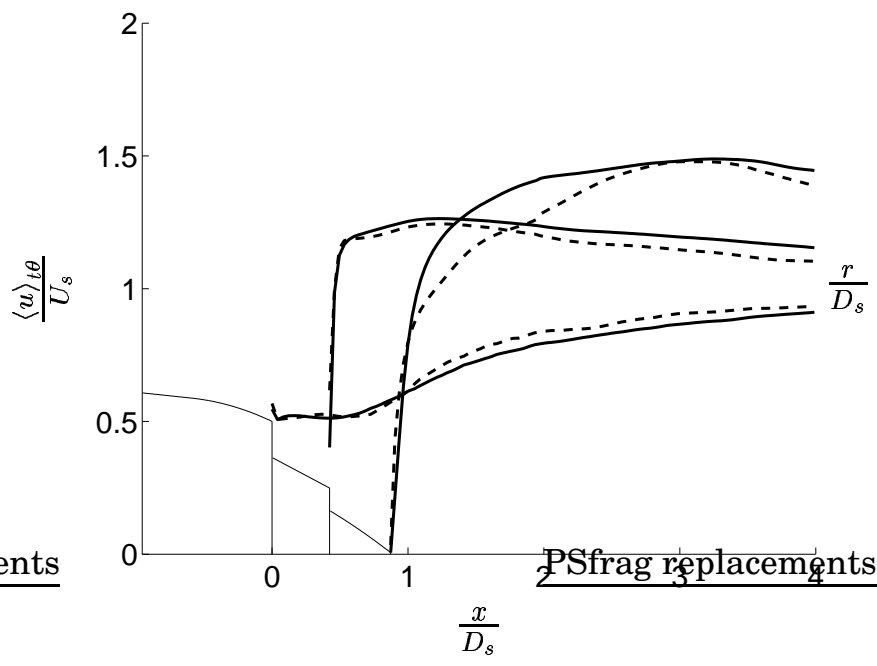

(a) Axial profiles

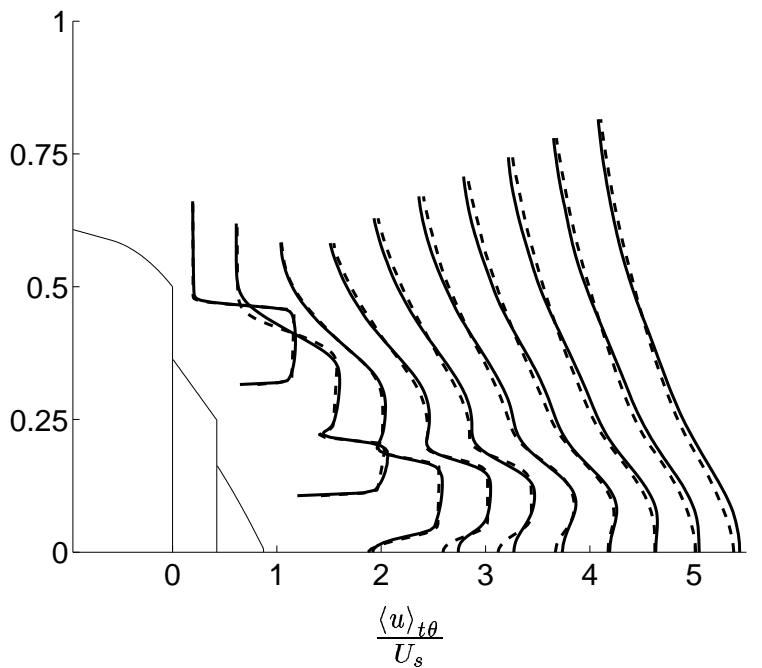

(b) Radial profiles

Figure 17. Axial and radial profiles of axial velocity. The profiles have been staggered according to their radial and axial location, respectively. Dashed lines denote case 1 and solid lines denote case 2.

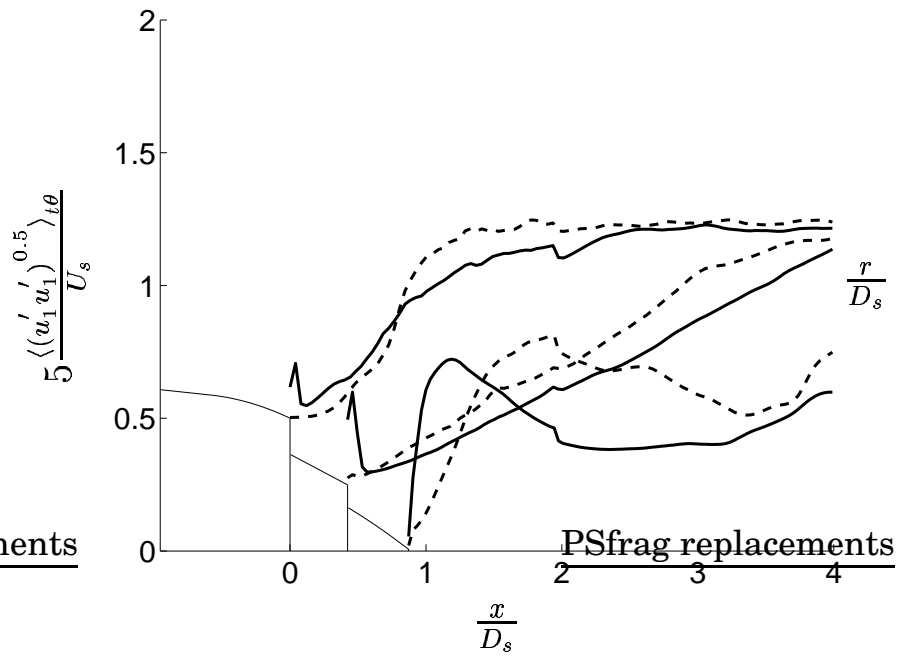

(a) Axial profiles

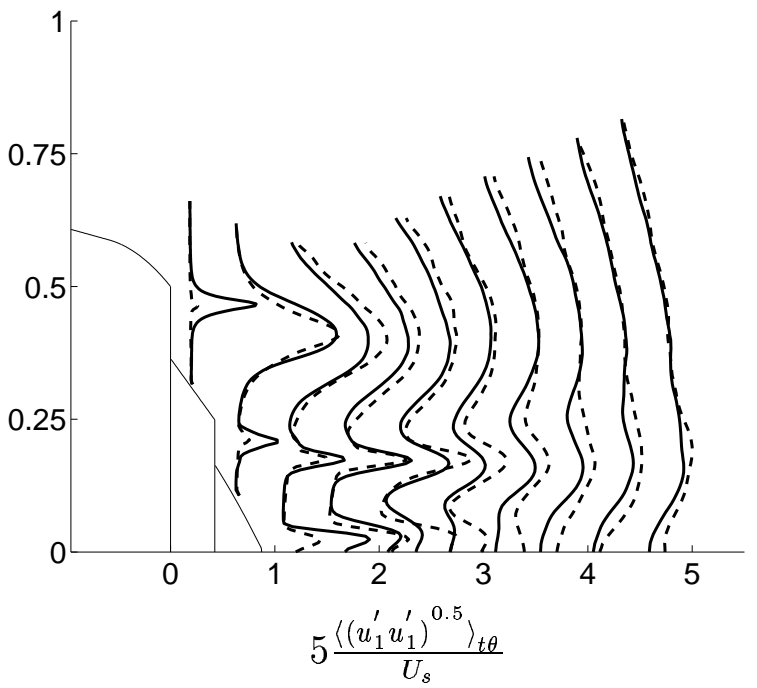

(b) Radial profiles

Figure 18. Axial and radial profiles of $u_{r m s}$. For legend see Figure (17). 


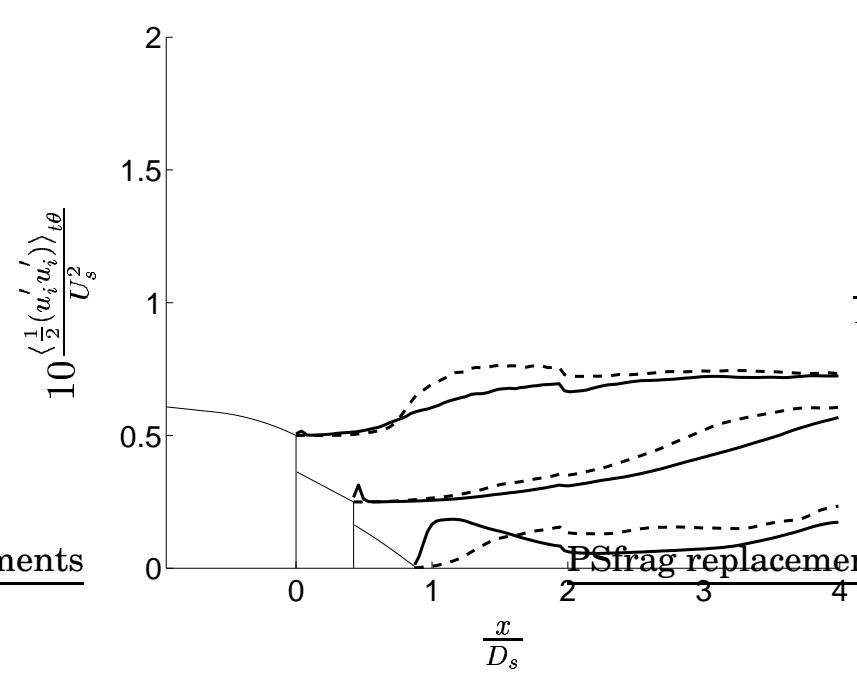

(a) Axial profiles

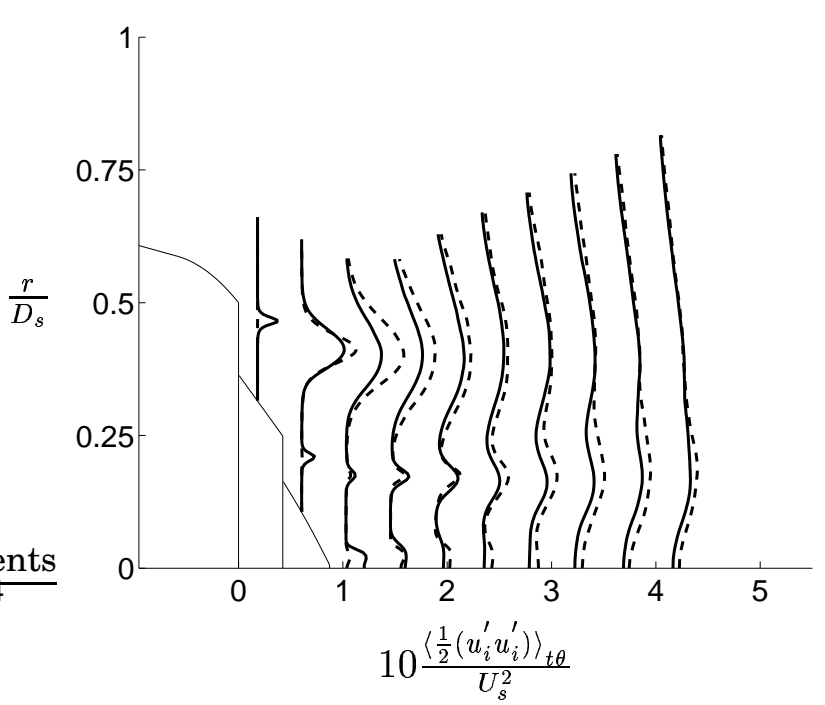

(b) Radial profiles

Figure 19. Axial and radial profiles of resolved turbulence kinetic energy. For legend see Figure (17).

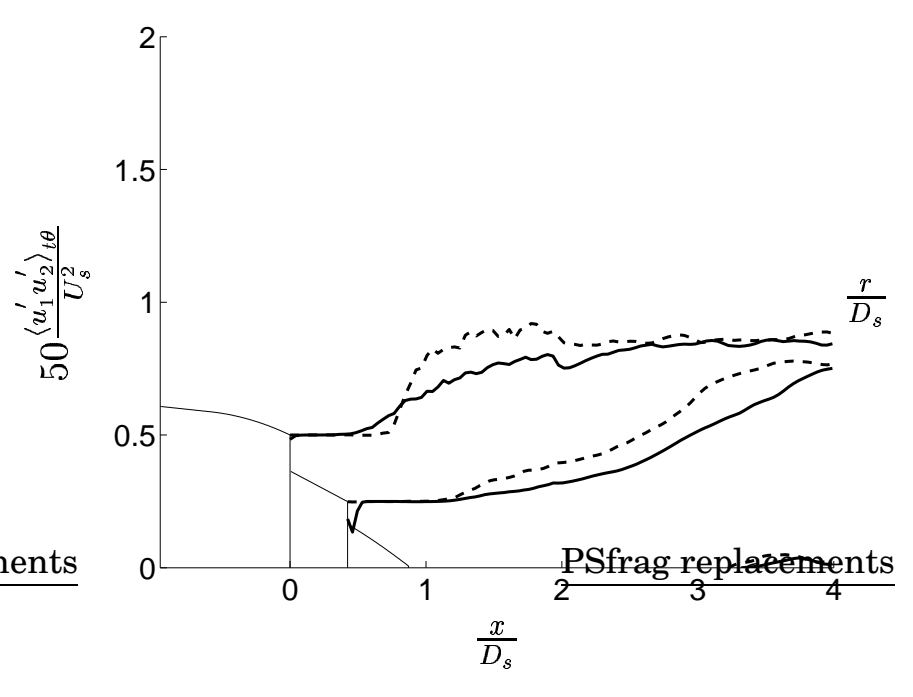

(a) Axial profiles

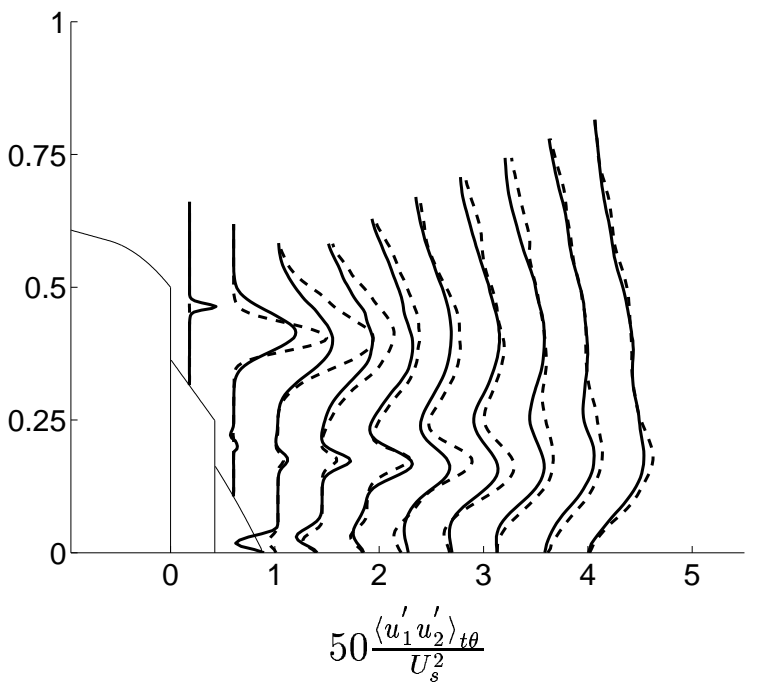

(b) Radial profiles

Figure 20. Axial and radial profiles of $u^{\prime} v^{\prime}$. For legend see Figure (17). 
where $u^{\prime}$ denotes resolved fluctuation of axial velocity and $\mathbf{x}$ is the position in the flowfield where the two-point correlation is evaluated. Figure (21) compares correlations obtained in the outer shear layer along the secondary nozzle lip line at an axial location corresponding to the downstream end of the center body. The spatial separation, $\xi$, is downstream in the axial direction. In total 26 correlation curves are represented in each of the figures. Each consecutive curve represents an increase of the spatial separation with $2.0[\mathrm{~mm}]$. As can be seen in the figures the correlations are wider in case 2 than in case 1 . This indicates that the turbulent flow in case 2 is closer to homogeneous turbulence than the flow in the case 1 simulation and that turbulence scales are larger in case 2 than in case 1.

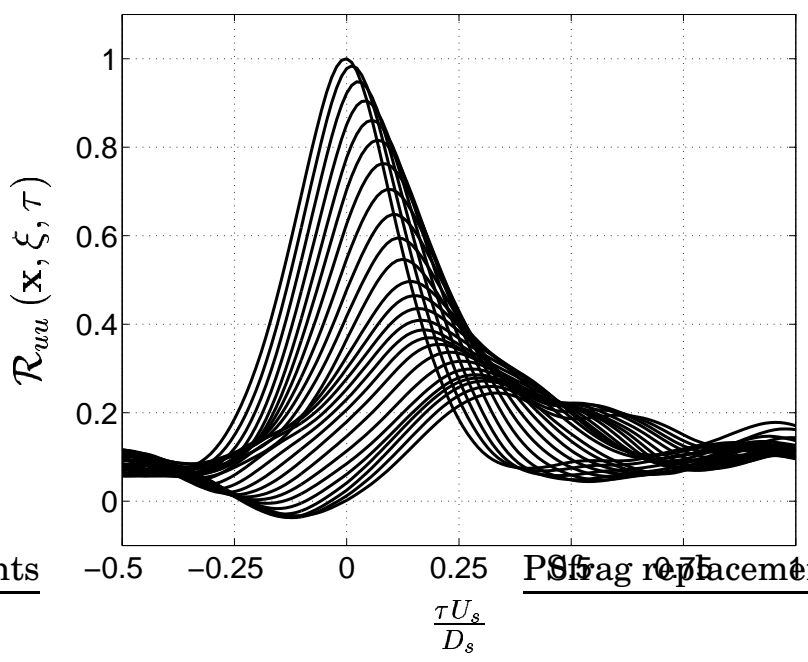

(a) case 1

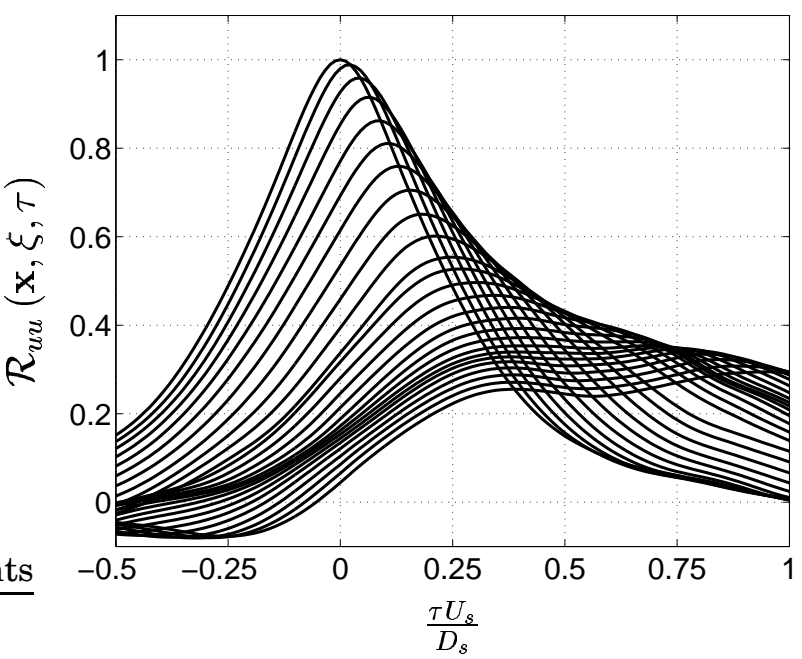

(b) case 2

Figure 21. Two-point space-time correlations obtained in the outer shear layer at an axial location corresponding to the tip of the center body, $\mathbf{x}=\left(x=0.25, r=0.5 D_{s}, 0\right)$.

\section{Far-field Sound Pressure Levels}

This section gives a presentation of predicted far-field sound pressure levels (SPL) and spectra of far-field pressure signals for case 1 and case 2. The far-field pressure signals were obtained using Kirchhoff surface integration. Sound pressure levels are presented in 90 observer locations. These observers are situated on two arcs, one centered on the centerline at the exit plane of the outer nozzle and one centered $1.5 \mathrm{~m}$ downstream of the center of the first arc, see Figure (2). The center of the second arc is approximately where the potential core ends in the RANS computations made for the same nozzle geometry and flow properties. On each of the two arcs there are 45 observer locations equally spaced between $20^{\circ}$ to $130^{\circ}$ from the $x$-axis for $\operatorname{arc} 1$ and from $30^{\circ}$ to $135^{\circ}$ degrees from the $x$ axis for arc 2. Figure (22) shows pressure signal power spectra for case 1 and case 2. Spectra obtained for four observer locations on the arc centered at the nozzle exit (arc 1) are presented in Figure (22(a)) and three observers located on the second arc (arc 2) are represented in Figure (22(b)). Note that the pressure signal time series obtained for case 1 is significantly shorter than the one obtained for case 2, which results in more uneven spectra in this case. The SPL presented in Figure (23) were obtained by integration of the power spectra from $S t=0.05$ up to a Strouhal number of 8.912 , which corresponds to 
a frequency of $10[\mathrm{kHz}]$. The figures show that the SPL is for almost all observer locations significantly higher in case 1 than in case 2. This is probably an effect of the high levels of turbulence kinetic energy found in the initial mixing region in case 1 . The fact that the sound pressure levels in case 1 are higher is most noticeable for the forward arc observers, which is natural since the forward arc is more affected by sound generated by small scale structures in the shear layers whereas the sound radiated to the rear arc observers is mainly generated by large structures in the region where the potential core ends. Consequently, the SPL peak is almost the same in the two cases but since the levels in the forward arc are higher in case 1 than in case 2, the sound directivity is not the same. There are at the moment no experimental data available for validation of the results, which means that there is no evidence of that the SPL obtained for case 2 are more correct than the ones obtained for case 1 . Nevertheless, it is believed that the flow obtained in the second simulation is more physically correct and that the corresponding predicted SPL therefore are more representative for the current nozzle/jet configuration. In either case, these results show the importance of predicting the flow in the initial flow region accurately in order to capture the acoustic signature of the jet.

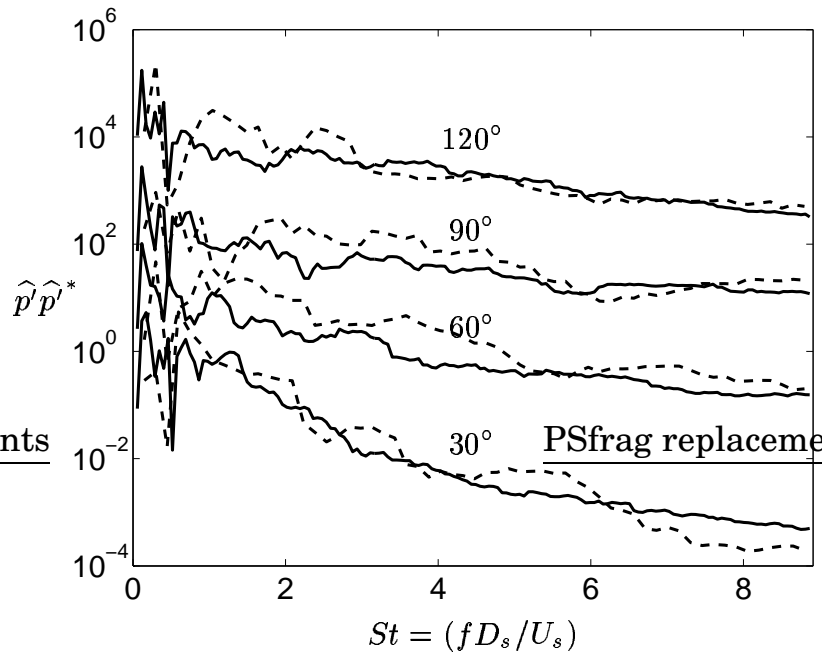

(a) $\operatorname{arc} 1$

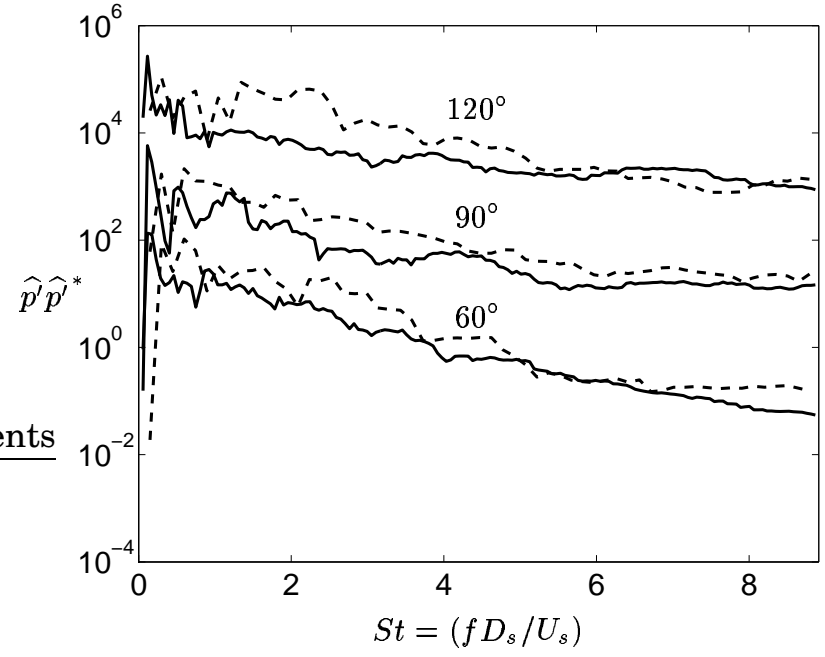

(b) $\operatorname{arc} 2$

Figure 22. Power spectra of far-field pressure signal for a few observer locations on the two arcs $\left(r=30 D_{s}\right)$. The spectra are band-pass filtered in third octave bands and have been staggered by multiplying the amplitude by a factor $10^{n}$, where $n=\left(\frac{\theta-30}{30}\right), \theta$ being the angle from the jet axis, see Figure (2). Dashed lines denote case 1 and solid lines denote case $2 . \widehat{p^{\prime}}$ denotes the Fourier transform of the pressure fluctuation and ${\widehat{p^{\prime}}}^{*}$ its conjugate.

\section{Concluding Remarks}

A high-subsonic coaxial jet has been simulated using large-eddy simulation. Two simulations have been made referred to in the paper as case 1 and case 2 , respectively. In the first simulation it was observed that the levels of mixing in the initial part of the outer shear layer were very low. This led to a rather violent mixing process once the shear layer broke up and hence the levels of turbulence kinetic energy were rather high in this region. Furthermore, it was found that the inner shear layer was very 


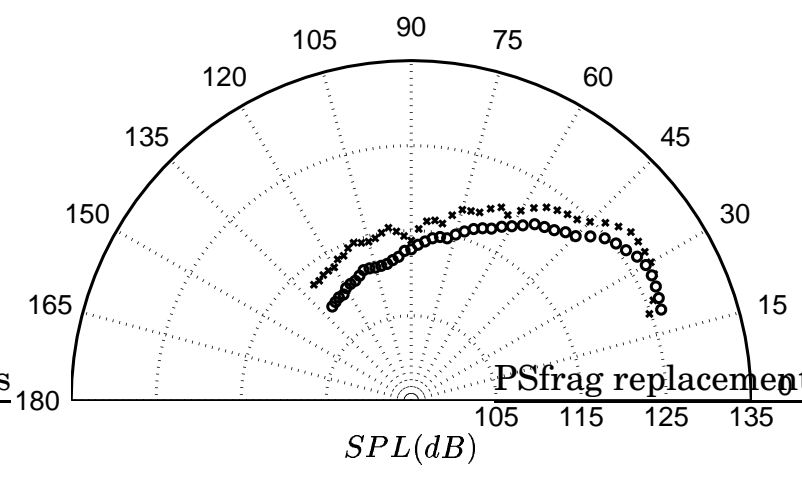

(a) $\operatorname{arc} 1$

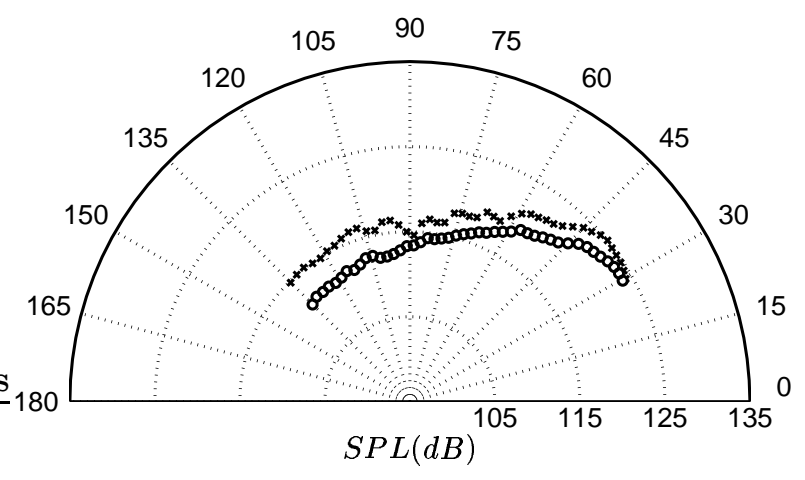

(b) $\operatorname{arc} 2$

Figure 23. Sound pressure levels in observer locations situated on a $30 D_{s}$ arc centered at the exit plane of the secondary nozzle (a) and on an arc centered at a location $1.5 \mathrm{~m}$ downstream of the previous location (b), see Figure (2). Predicted SPL are compared for case 1 (x-marks) and case 2 (circles).

thin and stable. Because of these observations a few changes were made in the simulation set-up. The subgrid-scale filter width, $\Delta$, was decreased from $\Delta=\left(\Delta_{1} \Delta_{2} \Delta_{3}\right)^{1 / 3}$ to $\Delta=\min \left(\Delta_{1}, \Delta_{2}, \Delta_{3}\right)$ with the objective of decreasing the SGS dissipation in the regions close to the nozzle and hence improving the resolved mixing in the outer shear layer. Furthermore, the accuracy of the scheme was improved by replacing the artificial selective damping needed to obtain stability in the simulation of case 1 to a TVD limiter for density in case 2. Owing to a decrease in numerical dissipation this further improved the mixing in the outer shear layer. Moreover, the shear layer between the core flow and the bypass flow became more unstable with this set-up. In both simulations total enthalpies and total pressures were specified at the nozzle inlet boundaries. However, in case 1 constant values were used whereas in case 2 the total pressures were specified as inlet profiles, which gave thicker boundary layers in the latter case.

For both simulations approximately $2.0 \times 10^{7}$ nodes were used for discretization of the calculation domain. In order to keep the number of cells down, the computational domain was divided into three regions: a well resolved near wall LES region, a medium-resolution LES region optimized for propagation of acoustic waves, and a coarse LES region. Over the interfaces between these regions, the number of cells is increased by a factor two in each direction. Special treatment of the interfaces between the regions based on cell reconstruction is utilized in order to minimize undesirable numerical errors.

The radial extent of the computational domain increases downstream such that the flow in the outer boundary region is sufficiently far from the hydrodynamic jet region and thus can be assumed to be irrotational and axisymmetric. Hence, the flow outside the three-dimensional computational domain can be represented by a less expensive two-dimensional axisymmetric calculation. Using this kind of boundary treatment enables the use of a rather narrow LES domain. Since the outer boundary of the $2 \mathrm{D}$ region is rather far from the jet the flow is not as affected of the boundary velocities specified at the boundary as if they were to be specified at the boundaries of the 3D domain. The interface between the full 3D LES region and the 2D region is based on azimuthally averaged quantities and acts as an absorbing boundary condition on both sides of the interface.

It should be noted that the simulations were made without increasing the viscosity or scaling down the geometry, which means that, although $2.0 \times 10^{7}$ nodes were used, it is a rather coarse LES. Wall functions were thus used to represent the near nozzle flow where needed.

High levels of turbulence kinetic energy found in the outer shear layer in case 1 resulted in signifi- 
cantly higher predicted sound pressure levels in case 1 than in case 2 . This result shows that changes in the initial mixing process have pronounced effects on the radiated sound and that it is thus important to capture the physics in the initial jet region to be able to predict the sound directivity. These effects are most noticeable for the forward arc observers. The peak sound pressure level was the same for both cases, which indicates that the initial mixing process is not as important for the rear arc observers as for the forward arc observers.

\section{Acknowledgment}

This work was conducted within the EU 6th Framework Project CoJeN (Computation of Coaxial Jet Noise), contract number: AST3-CT-2003-502790.

\section{References}

${ }^{1}$ Kirchhoff, G., "Zur Theorie der Lichtstrahlen,” Annalen der Physik und Chemie, Vol. 18, 1883, pp. 663-695.

${ }^{2}$ Ffowcs Williams, J. and Hawkings, D., "Sound Generated by Turbulence and Surfaces in Arbitrary Motion," Phil. Trans. Roy. Soc., Vol. A 264, 1969, pp. 321-342.

${ }^{3}$ Freund, J., Lele, S., and Moin, P., "Calculation of the Radiated Sound Field Using an Open Kirchhoff Surface," AIAA Journal, Vol. 34, No. 5, 1996, pp. 909-916.

${ }^{4}$ Uzun, A., 3-D Large-Eddy Simulation for Jet Aeroacoustics, Ph.D. thesis, School of Aeronautics and Astronautics, Purdue University, West Lafayette, IN, 2003.

${ }^{5}$ Uzun, A., Lyrintzis, A., and Blaisdell, G., "Coupling of Integral Acoustic Methods with LES for Jet Noise Prediction," International Journal of Aeroacoustics, Vol. 3, No. 4, 2005, pp. 297-346.

${ }^{6}$ Andersson, N., Eriksson, L.-E., and Davidson, L., "Investigation of an Isothermal Mach 0.75 Jet and its Radiated Sound Using Large-Eddy Simulation and Kirchhoff Surface Integration," International Journal of Heat and Fluid Flow, Vol. 26, No. 3 , 2005, pp. 393-410.

${ }^{7}$ Andersson, N., A Study of Mach 0.75 Jets and Their Radiated Sound Using Large-Eddy Simulation, Licentiate thesis, Division of Thermo and Fluid Dynamics, Chalmers University of Technology, Gothenburg, 2003.

${ }^{8}$ Andersson, N., Eriksson, L.-E., and Davidson, L., "Large-Eddy Simulation of Subsonic Turbulent Jets and Their Radiated Sound," AIAA Journal, Vol. 43, No. 9, 2005, pp. 1899-1912.

${ }^{9}$ Bogey, C., Bailly, C., and Juvé, D., "Computation of the Sound Radiated by a 3-D Jet Using Large Eddy Simulation," The 6th AIAA/CEAS Aeroacoustics Conference, No. 2009 in AIAA 2000, Lahaina, Hawaii, 2000.

${ }^{10}$ Bogey, C., Bailly, C., and Juvé, D., "Noise Investigation of a High Subsonic, Moderate Reynolds Number Jet Using a Compressible Large Eddy Simulation,” Theoret. Comput. Fluid Dynamics, Vol. 16, No. 4, 2003, pp. 273-297.

${ }^{11}$ Bogey, C., Bailly, C., and Juvé, D., "Noise Computation Using Lighthill's Equation with Inclusion of Mean Flow - Acoustic Interactions," The 7th AIAA/CEAS Aeroacoustics Conference, No. 2255 in AIAA 2001, Maastricht, Netherlands, 2001.

${ }^{12}$ Lighthill, M., "On Sound Generated Aerodynamically, I. General Theory," Proc. Roy. Soc., Vol. A 211, 1952, pp. 564-587.

${ }^{13}$ Bogey, C. and Bailly, C., "LES of a High Reynolds, High Subsonic Jet : Effects of the Inflow Conditions on Flow and Noise." The 9th AIAA/CEAS Aeroacoustics Conference, No. 3170 in AIAA 2003, Hilton Head, South Carolina, 2003.

${ }^{14}$ Bogey, C. and Bailly, C., "Investigation of Subsonic Jet Noise Using LES : Mach and Reynolds Number Effects." The 10th AIAA/CEAS Aeroacoustics Conference, No. 3023 in AIAA 2004, Manchester, United Kingdom, 2004.

${ }^{15}$ Bodony, D. and Lele, S., "Jet noise prediction of cold and hot subsonic jets using large-eddy simulation," The 10th AIAA/CEAS Aeroacoustics Conference, No. 3022 in AIAA 2004, Manchester, United Kingdom, 2004.

${ }^{16}$ Tanna, H., "An Experimental Study of Jet Noise Part I: Turbulent Mixing Noise," Journal of Sound and Vibration, Vol. 50, No. 3, 1977, pp. 405-428.

${ }^{17}$ Freund, J., "Noise Sources in a Low-Reynolds-number Turbulent Jet at Mach 0.9," Journal of Fluid Mechanics, Vol. 438, 2001, pp. 277-305.

${ }^{18}$ Shur, M., Spalart, P., Strelets, M., and Travin, A., "Towards the Prediction of Noise from Jet Engines," International Journal of Heat and Fluid Flow, Vol. 24, No. 4, 2003, pp. 551-561.

${ }^{19}$ Zhao, W., Frankel, S., and Mongeau, L., "Large Eddy Simulation of Sound Radiation from Subsonic Turbulent Jets," AIAA Journal, Vol. 39, No. 8, 2001, pp. 1469-1477.

${ }^{20}$ Erlebacher, G., Hussaini, M., Speziale, C., and Zang, T., "Toward the Large-Eddy Simulation of Compressible Turbulent Flows," Journal of Fluid Mechanics, Vol. 238, 1992, pp. 155-185.

${ }^{21}$ Crighton, D., "Basic Principles of Aerodynamic Noise Generation," Progress in Aerospace Sciences, Vol. 16 No. 1, 1975, pp. 31-96. 
${ }^{22}$ Lyrintzis, A., "Review: The Use of Kirchhoff's Method in Computational Aeroacoustics," ASME: Journal of Fluids Engineering, Vol. 116, 1994, pp. 665-676.

${ }^{23}$ Rahier, G., Prieur, J., Vuillot, F., Lupoglazoff, N., and Biancherin, A., "Investigation of Integral Surface Formulations for Acoustic Predictions of Hot Jets Starting from Unsteady Aerodynamic Simulations," The 9th AIAA/CEAS Aeroacoustics Conference, No. 3164 in AIAA 2003, Hilton Head, South Carolina, 2003.

${ }^{24}$ Eriksson, L.-E., "Development and Validation of Highly Modular Flow Solver Versions in G2DFLOW and G3DFLOW," Internal report 9970-1162, Volvo Aero Corporation, Sweden, 1995.

${ }^{25}$ Mårtensson, H., Eriksson, L.-E., and Albråten, P., "Numerical simulations of unsteady wakeflow," The 10th ISABE meeting, Nottingham, United Kingdom, 1991.

${ }^{26}$ Wollblad, C., Eriksson, L.-E., and Davidson, L., "Semi-implicit Preconditioning for Wall-bounded Flow," The 34th AIAA Fluid Dynamics Conference and Exhibit, No. 2135 in AIAA 2004, Portland, Oregon, 2004.

${ }^{27}$ Sagaut, P., Large Eddy Simulation for Incompressible Flows; an Introduction, Scientific Computation, Springer Verlag, 2nd ed., 2002, ISBN 3-540-43753-3. 